1 3D Electrohydrodynamic Printing of Highly Aligned Dual-Core Graphene

\title{
2 Composite Matrices
}

3 Baolin Wang ${ }^{1,2}$, Xing Chen ${ }^{1,2^{*}}$, Zeeshan Ahmad $^{3}$, Jie Huang ${ }^{4}$, Ming-Wei Chang ${ }^{1,2,5^{*}}$

$4{ }^{1}$ Key Laboratory for Biomedical Engineering of Education Ministry of China,

5 Zhejiang University, Hangzhou, 310027, P.R. China.

$6 \quad{ }^{2}$ Zhejiang Provincial Key Laboratory of Cardio-Cerebral Vascular Detection

7 Technology and Medical Effectiveness Appraisal, Zhejiang University, Hangzhou,

$8 \quad 310027$, P.R. China.

$9{ }^{3}$ Leicester School of Pharmacy, De Montfort University. The Gateway, Leicester, 10 LE1 9BH, UK.

$11{ }^{4}$ Department of Mechanical Engineering, University College London, London 12 WC1E7JE, UK.

$13{ }^{5}$ Nanotechnology and Integrated Bioengineering Centre, University of Ulster, 14 Jordanstown Campus, Newtownabbey, BT37 0QB, Northern Ireland, UK.

* Corresponding author: Ming-Wei Chang, Ph.D., Assoc. Professor Tel: +86(0)57187951517, Email: mwchang@zju.edu.cn

\begin{abstract}
The aim of this study was to develop an EHD printing method to fabricate grapheneloaded polycaprolactone (PCL)/polyethylene oxide (PEO) dual-core matrices. Graphene was incorporated in shell PCL components, while gelatin and dopamine hydrochloride (DAH) were encapsulated in two PEO cores to enhance biocompatibility of graphene-loaded matrices. Furthermore, the effect of PEO concentration on dualcore fiber formation was evaluated. The influence of process parameters (applied voltage, inner flow rate, outer flow rate and $X-Y-Z$ collector stage speed) on dual-core fiber morphology was evaluated. Our findings show graphene-loaded structures to possess two inner cores and increasing graphene content yields matrices with smoother surfaces, causing a slight reduction in their contact angle behavior. Furthermore, the
\end{abstract}


addition of graphene to matrices results in reduced elasticity. DAH release from matrices comprising various graphene concentrations showed no significant difference and drug release mechanism was diffusion based. In vitro biological tests indicate resulting graphene-loaded dual-core matrices exhibit good biocompatibility and also improve PC12 cell migration. The findings suggest matrices to have potential applications in nerve restoration and regeneration.

Keywords: graphene; dual-core; matrices; PC12 cells; EHD printing.

\section{Introduction}

Addressing peripheral nerve damage is an important challenge, in particular for reconstructive surgery, because of long-term disability and poor operative outcomes [1]. One approach to address loss of nerve tissue loss is to deploy suitable biomaterials such as artificial scaffolds [2]. Recent advances in tissue engineering include the development of artificial nerve grafts exhibiting similar physiological properties to peripheral nerves [3]. In other explorations, in vitro biological systems incorporate growth factors and physical stimuli (laser) have been used to guide artificial nerve conduits [4]. Numerous studies have focused on nerve guidance conduits consisting of intraluminal micro-channels with aligned fibers to improve nerve restoration [5].

One of the major factors involved in fabricating excellent nerve grafts is material selection. Polycaprolactone (PCL) is a hydrophobic polymer with desirable physicochemical properties including good biocompatibility, biodegradability and nontoxicity [6-8]. As shown previously, PCL has the ability to connect severely injured nerve stubs with significant mechanical properties [7]. Graphene is a single layer twodimensional $\mathrm{sp}^{2}$ carbon conductive nanomaterial in a honeycomb structure with unique physicochemical properties and has shown great potential in several biological applications [9]. For example, Graphene has been explored for anti-cancer/gene delivery, biosensing, biological imaging, antibacterial applications, cell culture and tissue engineering [10-12]. It has been previously reported that graphene improves cell 
bioelectricity between scaffold and cellular membrane because of $\pi$ bonding and large surface area [13]. For instance, it was found that three-dimensional graphene foams enhance neural stem cell growth and proliferation [14, 15]. Moreover, graphene demonstrates a high electron transfer velocity when compared to existing electronic materials [16]. Thus, it offers significant mechanical and biochemical cues for nerve restoration in tissue engineering.

Another factor often limiting the development of artificial nerve grafts is the engineering or fabrication method. The grafts should mimic the native extracellular matrix (ECM), both structurally and mechanically, in order to replicate the real neuro tissue environment [17]. Previous research has focused on the simulation of the natural ECM through the fabrication of fibrous constructs using various techniques [18-21]. However, emerging additive manufacturing methods, such as $3 \mathrm{D}$ printing permit the rapid and precise replication of tissue structures with micron-scale resolution [22]. Electrohydrodynamic (EHD) printing is an emerging 3D printing technique that allows flexibility in material selection and provides facile modulation of design parameters [23]. During the EHD process, the active ingredients can be incorporated on demand in a single step at room temperature. Thus, it is appropriate to generate versatile and anatomically accurate nerve grafts with a relatively high degree of reproducibility.

Complex fibers and particles have gained ample interest in recent years due to their potential in a wide range of biomedical applications [24]. For instance, hollow fibers have exceptional properties including low density, high specific surface area and tunable surface properties [25]. Besides, there is also an increasing need for the fabrication of core-shell fiber structures due to their potential in separating an unstable component or supporting a material to improve its mechanical properties [26]. Anisotropic fibers with multiple compartments can serve as drug carriers for controlled release of various drugs. Core-shell fibers, comprising distinct interior and exterior components, are promising structures for a broad range of applications, including filtration, tissue engineering, nanocomposites, smart textiles and green-energy 
applications [27-29]. In addition, these structures have been proposed for the strategic assembly and encapsulation of bioactive molecules [30]. The release rate of encapsulated biomolecules from the fiber core can be adjusted by carefully selecting shell material to manipulate bioactive release rate to be in sync with the promotion of sciatic nerve regeneration [31]. In addition, bio-active agents, with desired functional properties (e.g. antibacterial, conductivity [32-34]), can be encapsulated in tailored fashion (e.g. sub-micron location and release rate).

As for fiber alignment, a clear difference in fiber diameter distribution and film thickness across randomly orientated fibers is often observed [35]. Aligned fibers have potential applications in cell guidance (via release of cell-signaling moieties), supercapacitance and as biological scaffolds (possessing bioactive properties) where micron scaled structural arrangements are crucial for desirable biological functions and interactions [36, 37]. Furthermore, naturally occurring fibers constituting towards neural tissue are arranged in an anisotropic format, and the alignment of such fibers has been shown to facilitate cellular migration, proliferation, differentiation and maturation thus promoting tissue regeneration $[38,39]$. Moreover, for nerve restoration, direct suturing of nerve endings remains a challenge, and in this instance scaffolds with ordered fibers have shown to assist in neurite extension and axonal contact [38]. Combining properties of naturally occurring and synthetic polymers for fiber engineering and alignment can yield near-ideal conditions for specific bio-applications [40]. In this regard, the design and engineering of aligned and blended fibers is crucial to advance regenerative biomaterials $[41,42]$. Therefore, in this study, we developed and explored highly aligned dual-core graphene fiber matrices and their initial potential for nerve repair and regeneration.

Earlier studies have combined PCL and carbon based materials for similar applications [43]. Furthermore, reduced graphene oxide has been loaded into scaffolds for peripheral nerve injury repair, as shown by Vijayavenkataraman et al, where the EHD technique has been used to engineer 3D PCL/reduced graphene oxide scaffolds [44]. However, 
the potential to use graphene alongside more complex fiber structures (e.g. cores) was not explored.

This study focused on the design and fabrication of graphene loaded PCL nerve conduits using the EHD printing process. Despite graphene having a high electrical conductivity, the strong $\pi$ bond causes potential damage to the cell membrane integrity [45]. Therefore, it is necessary to modify graphene's surface to limit cytotoxicity. Dopamine (DA) is a neurotransmitter substance which has attracted considerable attention over the last few years, particularly within bio-sensing remits [46-48]. More specifically, the self-polymerization of DA, which yields polydopamine (PDA), has been explored as a multi-functional coating material [49]. Dopamine Hydrochloride (DAH) is the stable form of DA, whilst DAH is a key inotropic vasopressor agent which is closely associated with neurological diseases [50]. DAH has been explored as a potential functional replacement of DA [51, 52]. Dopamine hydrochloride (DAH) can enhance cell adhesion due to its high hydrophilicity, durable anti-erosion ability and well-documented biochemical properties [53, 54]. Thus in this study, DAH was used to improve biocompatibility of graphene loaded matrices. Gelatin is a natural biopolymer derived from the collagen, also has good hydrophilicity and cellular affinity [55]. To bring all these materials together and serve their specific purpose, an innovative method is devised to generate dual-core nerve grafts. Compared with traditional EHD printing fabrication, the technique applied in this research successfully overcomes many issues, such as integration of materials with different properties and low biocompatibility. In addition, the fabrication of well-ordered dual-core fiber matrices have not been reported previously.

Dual-core graphene matrices show good biocompatibility and improve PC12 cell migration. Results clearly indicate graphene-loaded dual-core matrices show potential applications in peripheral nerve restoration.

\section{Experimental}




\subsection{Materials}

Single-layered graphene (purity: over 98\%) was purchased from Suzhou Tanfeng Graphene Technology Co., Ltd. (China). Polyethylene oxide (PEO) (average molecular weight $9.0 \times 10^{5} \mathrm{~g} / \mathrm{mol}$ ) was obtained from Huagao Fine Chemical Co., Ltd. China. Polycaprolactone (PCL, mean $\mathrm{Mw}=8 \times 10^{4} \mathrm{~g} / \mathrm{mol}$ ) was purchased from Sigma-Aldrich, St Louis, USA. Dopamine hydrochloride (DAH) was provided by Aladdin Biochemical Technology Co., Ltd. China. Gelatin was purchased from Ourchem, China. Glacial acetic acid, absolute ethyl alcohol and phosphate buffer saline (PBS, $\mathrm{pH}=7.4$ ) were supplied by Sinopharm Chemical Reagent (China). Purified water was produced inhouse using a Millpore Milli-Q Reference ultra-pure water purifier (USA). All components were utilised as received without further purification. All chemicals and reagents used were of analytical grade.

\subsection{Solution Preparation}

Various concentrations of PEO solutions (in glacial acetic acid) were prepared to investigate polymer loading concentration effect on formation of dual-core fibers. PEO solutions with concentration of 2, 3, 4, 5 and $6 \% \mathrm{w} / \mathrm{w}$ were applied for both internal cores solutions during EHD printing process (the concentration of PEO solutions in two cores were kept identical). The mixtures were stirred for $7 \mathrm{~h}$ at a stable speed of $\sim 400$ rpm (VELP ARE, Italy) in order to prepare homogenous solutions. PEO solutions were dyed with $0.2 \%$ w/w Rhodamine B to allow differentiation between PEO and PCL layers under optical microscopy. Subsequently, one PEO solution with appropriate concentration was selected for the next steps. Subsequently, 5\% w/w DAH and 50\% w/w gelatin (of PEO quantity) were added to PEO solutions, respectively, to serve as two different inner cores.

PCL solutions were utilised for the shell layer of dual-core matrices in all experiments. PCL pellets were suspended in glacial acetic acid (concentration 24\% w/w) and were stirred for $5 \mathrm{~h} \sim 150 \mathrm{rpm}$ to ensure complete dissolution (VELP ARE, Italy). Graphene was added to PCL solutions at various concentrations $(0.05 \%, 0.1 \%, 0.2 \%$ and $0.3 \%$ 
w/w (of PCL quantity)) and were placed under mild bath sonication for $1 \mathrm{~h}$. The solutions were under continuous stirring for $1 \mathrm{~h}$ to form homogenous solutions. All experiments were conducted at the ambient temperature $\left(25^{\circ} \mathrm{C}\right)$.

\subsection{Graphene loaded dual-core matrices fabrication}

A dual-core nozzle assisted EHD printing system was used for the fabrication of the matrices. The schematic of the process is shown in Fig. 1(a). The main components of this system are the high voltage supply (DC, Glassman high voltage Inc. series FC, USA), a high precision $X-Y-Z$ moving stage along with the controller (Hongxia automatic control equipment Co., Guangzhou, China), and three syringe pumps (KD Scientific KDS100, USA). PCL and the two PEO solutions were loaded separately into three $5 \mathrm{~mL}$ syringes; each controlled by its own infusing syringe pump. A dual-core nozzle (outer diameter for the shell was $4 \mathrm{~mm}$ and outer diameter for the two core were both $1 \mathrm{~mm}$, and the distance between two cores was $0.1 \mathrm{~mm}$ ) was placed on the Z-axis and connected to the positive port of the voltage supply. Two inner cores of the nozzle were connected to two separate syringes loaded with PEO solutions via silicon tubing. The outer shell media (nozzle) was infused with the PCL solution. The glass substrate served as the collecting point and was mounted on grounded a $X$-Y moving staging. The working principle of this EHD printing process is based on the balance between the electrostatic force, the combined surface tension and the viscoelastic force of the liquid [56]. To enable this process, all solutions were subjected to a high and sustained voltage of identical value. The movement of $X-Y-Z$ stage could easily control deposition and shapes of dual-core matrices. The stage controlling system used for the EHD printing process was purchased from Zhongweixing Technology Co., LTD (China). This allows input of co-ordinate based patterns (e.g. X, Y and Z data) into the system. STL files cannot be directly fed into the software. Other file formats such as Hewlett Packard Graphic Languages (HPGL/PLT) can be directly transferred to the software to control stage movement.

The substrate collecting speed was varied between $20-100 \mathrm{~mm} / \mathrm{s}$. The high voltage 
supply applied between the nozzle and the substrate was in the order of 2.3-2.8 KV with the working distance at $5.0 \mathrm{~mm}$. Flow rate of PCL solution was within the range of 0.25-0.5 ml/h. For PEO solutions (two internal cores), flow rate was varied between $0.12-0.22 \mathrm{ml} / \mathrm{h}$. The fabrication process involved the patterning of the complex $3 \mathrm{D}$ graphene loaded dual-core matrices with accurate overwriting of fiber layers (20 layers in total with 10 layers along X-axis and 10 layers along Y-axis). All experiments were conducted at ambient temperature $\left(25^{\circ} \mathrm{C}\right)$ and relative humidity $(40-60 \%)$.

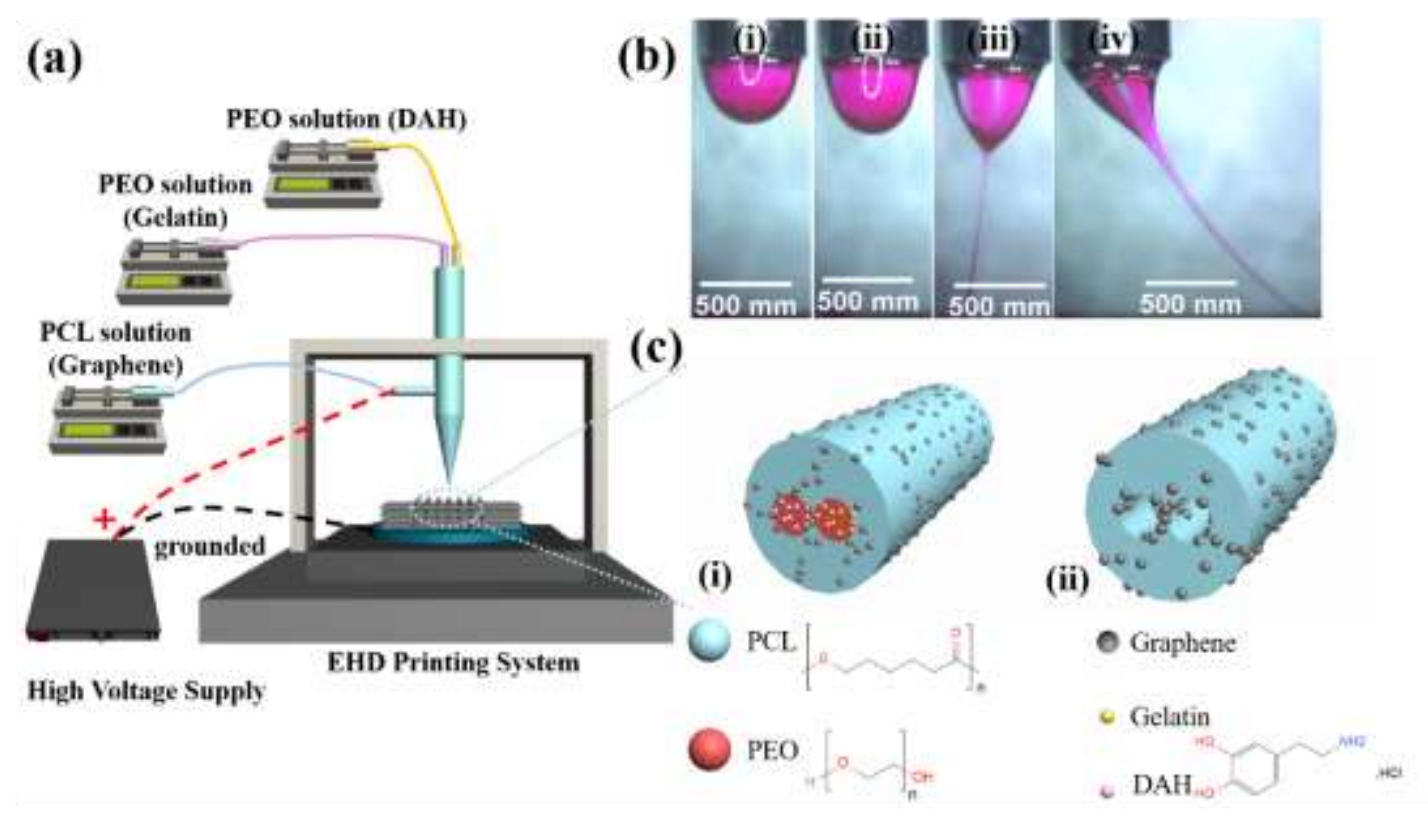

Fig. 1. (a) Schematic of the dual-core EHD printing system. (b) Process images of dualcore jet during the formation of dual-core filaments. (c) Schematic diagram of graphene loaded dual-core fibers (i) before and (ii) after the drug release process.

\subsection{Characterization of graphene loaded dual-core matrices}

Graphene loaded dual-core matrices were examined using Scanning Electron Microscopy (SEM, FEI Quanta 650, Netherland) for 3D structure evaluation. All samples were placed on an aluminum stub with double-sided conductive tape and were coated with a thin layer of platinum for 90s by sputter coating (1080auto, Cressington Scientific Instruments Ltd., UK) at the current intensity at $25 \mathrm{~mA}$. Optical microscopy (OM, Phoenix BMX503-ICCF, China) was employed in this research to image specific 
inner structures of matrices. Mean fiber diameters were calculated using 50 random fibers for each sample set. Graphene appears as dark-black regions under optical imaging, and graphene loaded dual-core matrices would show a deeper contrast at higher concentration of the material. In this study, all data was exported for analysis and was plotted as graphs via Origin software (OriginLab, USA). Error bars in the graphs denote mean \pm standard deviation.

\subsection{Contact Angle Measurement}

Water contact angle represents the level of hydrophilicity or hydrophobicity of matrices. Structure contact angle and interfacial tension analysis (SL2000KB, Kino Industry Co., Ltd, USA) was measured in this study. The contact angle of one PBS droplet ( $1 \mu \mathrm{L})$ on the dual-core matrices from point of contact to 5 min was investigated.

\subsection{Fourier Transform Infrared (FTIR) and X-ray Diffraction (XRD) Spectroscopy}

Fourier Transform Infrared (FTIR) spectra for graphene loaded dual-core matrices were recorded (IR Affinity $1\left(4000-500 \mathrm{~cm}^{-1}\right)$, Shimadzu, Japan) to investigate composition and material stability. Samples were prepared using the $\mathrm{KBr}$ pellet pressing method. In short, $2 \mathrm{mg}$ of printed samples were mixed with $200 \mathrm{mg} \mathrm{KBr}$ medium by grinding. Mixtures were compressed into transparent pellets under a force of $12 \mathrm{MPa}$. FTIR spectra were acquired following 20 scans. Wide-angle X-ray diffraction (XRD) patterns of graphene loaded dual-core matrices were recorded in this study using an X-ray crystal diffractometer (Gemini A Ohra, Oxford, UK). The specimens were scanned over a 2-theta range of $5^{\circ}-60^{\circ}$ at a step size of $0.02^{\circ}$ at $40 \mathrm{KV}$ and $40 \mathrm{~mA}$.

\subsection{Mechanical Testing}

Tensile properties of dual-core matrices were investigated using a universal materials tester (Roell Z020, Zwick, Germany). A load cell of $500 \mathrm{~N}$ was applied. Test specimens were rectangular grid-shape matrices of length $30 \mathrm{~mm}$ and width $10 \mathrm{~mm}$ (the thickness was $\sim 100 \mu \mathrm{m}$ ). Tests were conducted at a strain rate of $10 \mathrm{~mm} / \mathrm{min}$ at ambient conditions. For each sample set, tests were conducted three times after which the mean elastic 
modulus was calculated.

\subsection{Drug Release from Dual-core Matrices}

Drug release behavior of DAH from graphene loaded dual-core matrices was analyzed according to a method reported previously [57]. Assays to be applied in release tests comprised $10 \mathrm{ml}$ release medium (PBS) with $30 \mathrm{mg}$ of dual-core matrices for each sample. Samples were vibrated $(200 \mathrm{rpm})$ at temperature of $37{ }^{\circ} \mathrm{C}$ in a $\mathrm{HZ}-8801 \mathrm{~K}$ thermostatic oscillator (Taicang Science and Education Factory, China). During drug release period, $3 \mathrm{ml}$ of supernatant was removed for UV detection and replaced with an identical volume of fresh release medium at preset time intervals. The concentration of DAH in the supernatant was investigated by UV absorption at a wavelength of $280 \mathrm{~nm}$ (UV-2600 spectrophotometer, Shimadzu, Japan). Each experiment was conducted in triplicate.

In this study, Korsmeyer-Peppas and Higuchi curve-fitting models were applied to analyze drug release kinetics of DAH release. The Korsmeyer-Peppas model is generally used if drug release mechanism is not clear. This model can be presented as equation (1):

$$
\frac{M_{t}}{M_{\infty}}=k t^{n}
$$

Here, $M_{t}$ is the cumulative quantity of drug released at time t, and $M_{\infty}$ is the initial drug loading, while $\mathrm{k}$ is a constant and $\mathrm{n}$ is the release exponent indicating the release mechanism [58]. The Higuchi model is normally used to confirm diffusive drug release from a polymer matrix system, and it can be presented as shown in equation (2):

$$
M_{t}=k_{H} t^{\frac{1}{2}}
$$

Here, $M_{t}$ is the quantity of cumulative drug release after time $\mathrm{t}$ and $k_{H}$ is the Higuchi constant.

\subsection{PC12 Cell Culture}

PC12 cell line is derived from pheochromocytoma of the rat adrenal medulla. These 
neuronal fibroblasts-like cells are usually used for nerve implant studies [59, 60]. Cells were routinely cultured in Dulbecco's modified Eagle's medium (DMEM) (Coring, USA), supplemented with 10\% fetal bovine serum (FBS) (Sijiqing, China), 5\% horse serum (Sijiqing, China), and 1\% antibiotic antimycotic solution (Biyuntian, China) at $37^{\circ} \mathrm{C}$ in humidified atmosphere of $5 \% \mathrm{CO}_{2}$. The culture medium was refreshed every 2 days. All samples used in cell tests were sterilized under UV light for $24 \mathrm{~h}$ and then fixed with sterilized stainless-steel rings in the culture dish.

\subsection{CCK-8 Cell Viability test}

$100 \mu \mathrm{L}$ of PC12 cell suspension was placed into a 96 -well plate at the density of $1 \times 10^{4}$ cells/well and was incubated for $24 \mathrm{~h}$. CCK-8 tests was conducted to investigate proliferation of PC12 cells on four various graphene loaded dual-core matrices and $0.11 \%$ w/w graphene loaded matrices without DAH as well as gelatin. The samples were cut into discs (diameter $=6 \mathrm{~mm}$ ) and sterilized under UV light for $24 \mathrm{~h}$ prior to being added to the culture plate. Following incubation for 3 days, cell viability was measured by adding $10 \mu \mathrm{L}$ CCK-8 solution to each well and incubated for a further $4 \mathrm{~h}$. Absorbance was measured at a wavelength of $450 \mathrm{~nm}$ using a microplate reader (Multiskan GO, Thermo Fisher Scientific, USA). The control group was cells cultured on plain TCP wells, and the blank group was culture medium with CCK-8 solution. The relative cell viability (\%) was counted by equation (3):

Cell viability $(\%)=\frac{A b \cdot(\text { sample })-A b \cdot(\text { blank })}{A b .(\text { control })-A b .(\text { blank })} \times 100 \%$

Where $\mathrm{Ab}$. represents the absorbance.

\subsection{Cell Morphology Study}

Morphology of cells seeded on scaffolds was evaluated by fluorescent microscopy. PC12 cells cultured on matrices were fixed with $4 \% \mathrm{v} / \mathrm{v}$ formalin for $20 \mathrm{~min}$ at the ambient temperature $\left(25^{\circ} \mathrm{C}\right)$, and were washed 3 times using PBS $(\mathrm{pH}=7.4)$. Cells were permeable with $0.1 \%$ Triton X-100 in PBS for 5 min followed by washing with 
PBS. Subsequently, cell cytoskeleton and nuclei were stained with Alexar Fluor 546 phalloidin (Yeasen Biology Technology Co., Ltd, China) (1:100 dilution) and 4',6'diamidino-2-phenylindole hydrochloride (DAPI, Yeasen Biology Technology Co., LTD, China) for 20 and $5 \mathrm{~min}$, respectively. Specimens were washed a further three times with PBS after each staining step. Finally, PC12 cells were observed using an inverted fluorescent microscope (Nikon, Eclipse Ti-S, Japan).

\subsection{Cell Migration Assay}

In vitro scratch assays were applied in this study to investigate the influence of graphene on PC12 cell migration. Dual-core matrices were sterilized as shown previously. PC12 cells were seeded in a 6 -well TCP plate at a density of $3 \times 10^{5}$ cells/well and incubated at $37^{\circ} \mathrm{C}$ in $5 \% \mathrm{CO}_{2}$ humidified atmosphere, until cells were confluent and the formation of monolayers was apparent. A $200 \mu \mathrm{L}$ sterile pipette tip was used to make a scratch on the cell monolayer surface which was then washed with PBS gently for the removal of free cells. Fresh culture medium was added to the scratched specimen well and cells were incubated at $37{ }^{\circ} \mathrm{C}$ and $5 \% \mathrm{CO}_{2}$ in a humidified atmosphere. Images were taken at 0, 24 and 72h using an inverted fluorescent microscope (Nikon, Eclipse Ti-S, Japan) to observe cell migration on the scratched surface.

\subsection{Statistical Analysis}

All tests were repeated three times and results were displayed as mean \pm standard deviation. The $p$ value of 0.05 was considered significant using a one-way ANOVA test.

\section{Results and Discussion}

\subsection{Fabrication and characterization of graphene loaded dual-core matrices}

In this study, a dual-core nozzle assisted EHD printing method was used to fabricate 3D graphene loaded dual-core matrices. Figs. 1(a) and (b) display images of the dualcore jetting process during the formation of matrices. Rhodamine $\mathrm{B}$ was used to differentiate between PEO and PCL layers within the matrices; resulting in a red PEO 
layer. A droplet with a red inner core can be observed hanging at the tip of nozzle before high voltage was applied to the nozzle and the color of the droplet indicates both PCL solution and PEO solution were present. Fig. 1(bii) shows that increasing the applied voltage to $1.8 \mathrm{kV}$ (causing an increase in electric field intensity) resulted in the droplet stretching under electrical force and gravity. When the applied voltage reached $2.2 \mathrm{kV}$, the liquid jet deforms further as shown in Fig. 1 (biii). Here, the two inner fluids can be observed clearly. Based on this condition, the EHD printing jet is stretched with the movement of $X$-Y stage (Fig. 1(biv)). Fig. 1(c) shows a schematic diagram of graphene loaded dual-core fibers. Graphene was loaded into the outer PCL layer, while DAH and gelatin were encapsulated in two inner PEO cores separately (Fig. 1(ci)). As PEO is soluble in DI water, the dual-core fibers would show two hollow channels after being immersed in DI water as shown in Fig. 1(cii).

\subsection{Effect of PEO Concentration on Morphology of Dual-core matrices}

According to previous studies, polymer concentration has significant impact on morphology of sub-micron fibers [61]. In this research, the concentration of PCL was chosen based on preliminary experimentations using concentrations between $20 \%$ and $25 \% \mathrm{w} / \mathrm{w}$ [62]. PEO solutions have been used for the EHD printing process [63], however, there are no studies making its use as the inner core component for the formation of dual-core fibers. In this study, various concentrations of PEO solutions were used to investigate its effect on the morphology of the dual-cores fibers. Fig. 2(a) exhibits optical image of the resulting dual-core fibers generated when the inner PEO solution loading was fixed to $2 \% \mathrm{w} / \mathrm{w}$. The applied voltage was set at $2.6 \mathrm{kV}$ and the flow rate of the PCL solution was set to $0.35 \mathrm{~mL} / \mathrm{h}$. The flow rates of the two inner PEO solutions was constant at $0.15 \mathrm{~mL} / \mathrm{h}$. Two continuous inner core fibers were found in all filaments, indicating dual-core structures were successfully fabricated, whilst also demonstrating high reproducibility. It can also be observed that the size of the two internal PEO fibers were near identical within dual-core filament. Fig. 2(b) shows that increasing PEO concentration from 2 to $5 \% \mathrm{w} / \mathrm{w}$ did not have a major impact on the dual-core fiber diameter. As for PEO concentration of $6 \% \mathrm{w} / \mathrm{w}$, the overall fiber 
diameter was lower than fibers fabricated using 5\% w/w PEO, with a higher standard deviation, indicating dual-core fiber uniformity was uneven at these conditions.

The diameter of single PEO fibers increased with an increase in concentration from $2 \%$ to $5 \% \mathrm{w} / \mathrm{w}$. However, increasing PEO concentration to $6 \% \mathrm{w} / \mathrm{w}$ leads to a decrease in inner PEO diameter (Fig. 2(c)). This may be due to the high viscosity of the liquid impeding flow at the inner core nozzle. The core/fiber ratio is the ratio of a single core to the overall fiber diameter and is shown in Fig. 2(d). The core ratio was enhanced when PEO concentration is increased from 2 to 5\% (Fig. 2(d)). For dual-core fibers produced using $6 \% \mathrm{w} / \mathrm{w}$ PEO concentration, the mean value of core ratio was similar to that of $5 \% \mathrm{w} / \mathrm{w}$ concentration. Therefore, to maximize the function of inner components and obtain dual-core structures with good morphology, 5\% w/w PEO concentration was chosen for subsequent experiments.
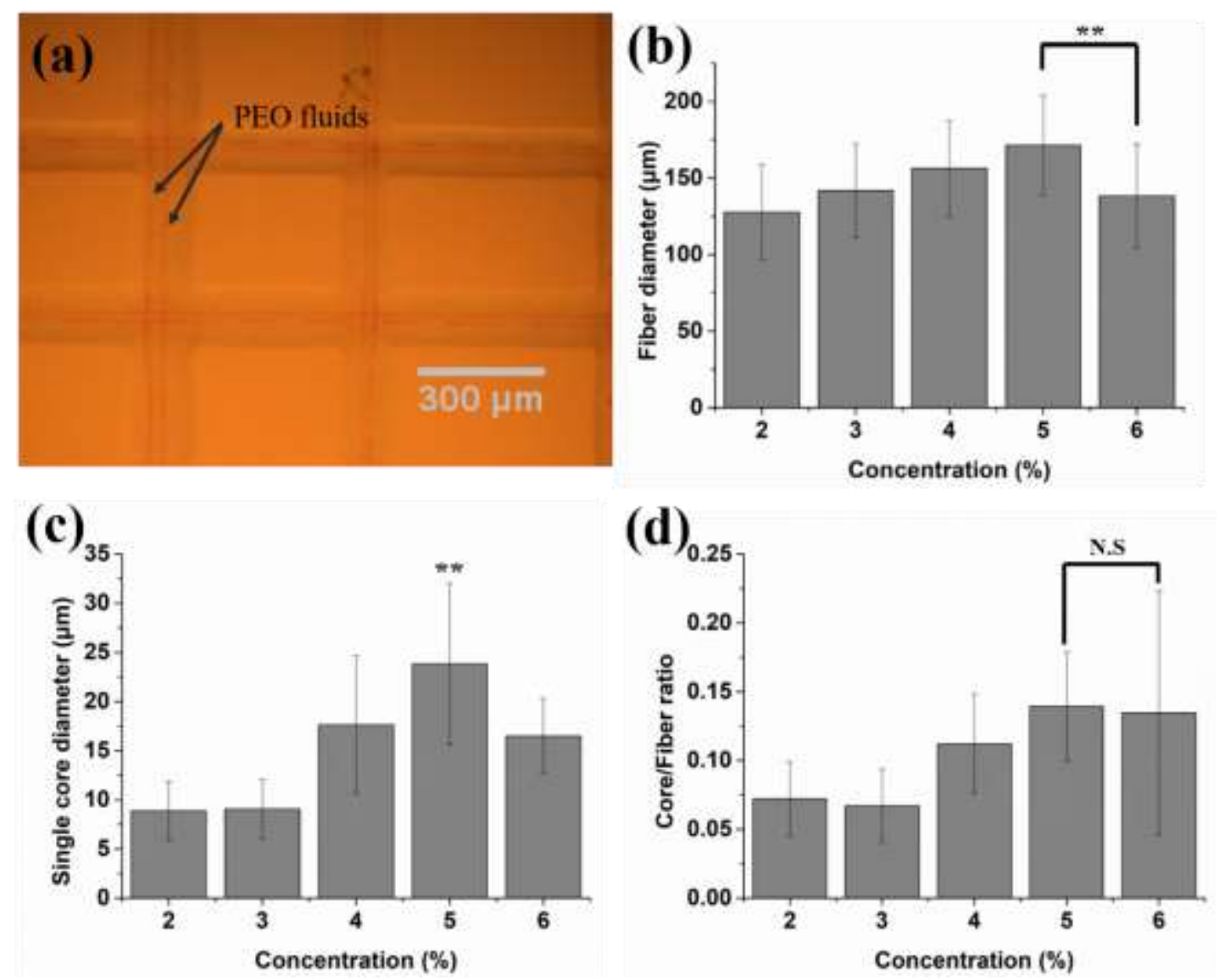

Fig. 2. (a) Optical microscopy images of dual-core fibers when the PEO concentrations was $2 \% \mathrm{w} / \mathrm{w}$. This image was obtained under the moving speed of $X-Y-Z$ stage at 60 
$\mathrm{mm} / \mathrm{s}$. (b) Fiber diameter, (c) single core diameter and (d) core/fiber ratio changed with increasing PEO concentration. (** represents significant difference, $p<0.01 ;$ N.S. represents there was no significant difference.)

EHD process parameters have a significant influence on the resulting fiber diameter and matrix morphology. Some studies have investigated the effects of these parameters on fiber morphology [56], however, research on the effect of process parameters on dual-core fibers via EHD printing is scarce. In this research, the distance between nozzle tip and collecting substrate was kept at $5 \mathrm{~mm}$ for all studies. The dual-core nozzle for EHD printing process is not stable especially when varying the working distance (tip to substrate). The working distance for a stable Taylor-cone is very narrow, and the fiber diameter did not show significant difference within this range. Therefore, in order to achieve stable printing, the work distance was kept same for all experiments. In this study, applied voltage, synchronous two inner flow rate, outer flow rate for PCL solution, and moving speed of $X-Y-Z$ stage were varied and their effect on dual-core fiber diameter was studied as shown in Fig. 3. The applied voltage was varied from 2.3 up to $2.8 \mathrm{kV}$ at increments of $0.1 \mathrm{kV}$. Dual-core fiber diameter firstly decreased from 110 to $100 \mu \mathrm{m}$ as the voltage increased from 2.3 to $2.6 \mathrm{kV}$, then the fiber diameter increased to $130 \mu \mathrm{m}$ when the voltage was $2.8 \mathrm{kV}$, as shown in Fig. 3(a). Changing the two inner PEO flow rates simultaneously from $0.12 \mathrm{~mL} / \mathrm{h}$ to $0.22 \mathrm{~mL} / \mathrm{h}$ significantly improved the dual-core fiber diameter from 75 to $156 \mu \mathrm{m}$, which is presented in Fig. 3(b). Fig. 3(c) shows the fiber diameter varying with outer flow rate from $0.25 \mathrm{~mL} / \mathrm{h}$ to $0.50 \mathrm{~mL} / \mathrm{h}$. It was found that increasing outer flow rate resulted in an increase in fiber diameter. When the collecting $X-Y-Z$ stage speed increased from 20 to $100 \mathrm{~mm} / \mathrm{s}$, the fiber diameter of dual-core fibers dramatically reduced from 130 to $70 \mu \mathrm{m}$ (Fig. 3(d)). This is because the fibers undertake high drawing force upon increase of moving speed. 

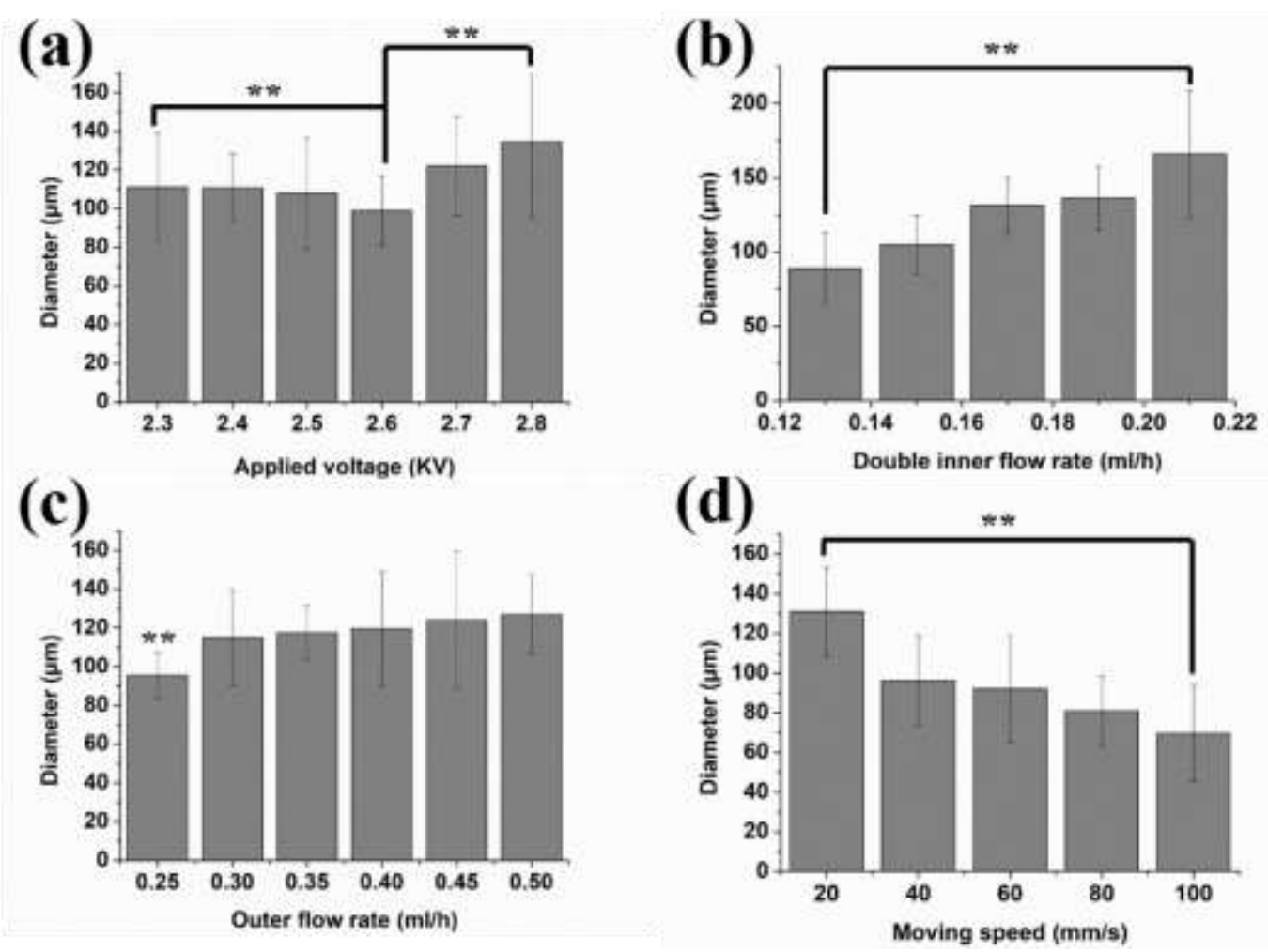

419 Fig. 3. (a) Effect of applied voltage on dual-core fiber diameter (moving speed $=60$ $\mathrm{mm} / \mathrm{s}$, inner flow rate $=0.15 \mathrm{ml} / \mathrm{h}$, outer flow rate $=0.35 \mathrm{ml} / \mathrm{h}$ ). (b) Effect of changing two inner flow rate simultaneously on dual-core fiber diameter (moving speed $=60$ $\mathrm{mm} / \mathrm{s}$, applied voltage $=2.6 \mathrm{KV}$, outer flow rate $=0.35 \mathrm{ml} / \mathrm{h}$ ). (c) Effect of outer flow rate on fiber diameter (moving speed $=60 \mathrm{~mm} / \mathrm{s}$, inner flow rate $=0.15 \mathrm{ml} / \mathrm{h}$, applied voltage $=2.6 \mathrm{KV}) .(\mathrm{d})$ Effect of moving speed of $X-Y-Z$ stage on fiber diameter (applied voltage $=2.6 \mathrm{KV}$, inner flow rate $=0.15 \mathrm{ml} / \mathrm{h}$, outer flow rate $=0.35 \mathrm{ml} / \mathrm{h}) .(* *$ represents significant difference, $p<0.01$ )

\subsection{Effect of Graphene Concentration on Morphology of Dual-core Matrices} Fig. 4(a) shows digital images of the dual-core matrices loaded with various concentrations of graphene. Evidently the more graphene that was loaded into the matrices, the darker the appearance of the final product. Fig. 4 also displays SEM images of graphene loaded matrices at different magnifications. The surface morphology of dual-core matrices loaded with various graphene concentration is

434 illustrated in Figs. 4(b-f). Fig. 4(b) is the image of dual-core matrices without graphene; 
showing a porous surface which is comparable to pure PCL fiber morphology reported previously [57]. The addition of a small amount of graphene $(0.05 \% \mathrm{w} / \mathrm{w}$ and $0.1 \%$ w/w) causes matrix surface to become uneven but without any pores (Fig. 4(c) and 4(d)). When the concentration of graphene was continuously increased to $0.3 \% \mathrm{w} / \mathrm{w}$, the dualcore matrices developed smoother surfaces, as shown in Fig. 4(e) and 4(f). These results indicate that loading graphene into the PCL shell layer has a significant influence on matrix morphology and surface texture. The overall structures of all dual-core matrices were similar as shown in Fig. 4(g). The matrices were well-ordered, and stacked at high precision. The width of each grid within the matrices was $500 \mu \mathrm{m}$. It was also found that each layer was clearly distinguishable (Fig. 4(h)). Fig. 4(i) is a cross-section SEM image of dual-core fibers after immersion in DI water. As PEO is soluble in water, two hollow channels are formed, indicating dual-core fibers were generated successfully. In addition, fiber diameter of matrices shown in Fig. 4 was not within the range shown in Fig. 3, which is due to the first printed layer exhibiting a flat morphology rather than cylindrical shape. This is due to an increased contact area with the collecting substrate. Fig. 3 shows the data measured by the first printing layer.

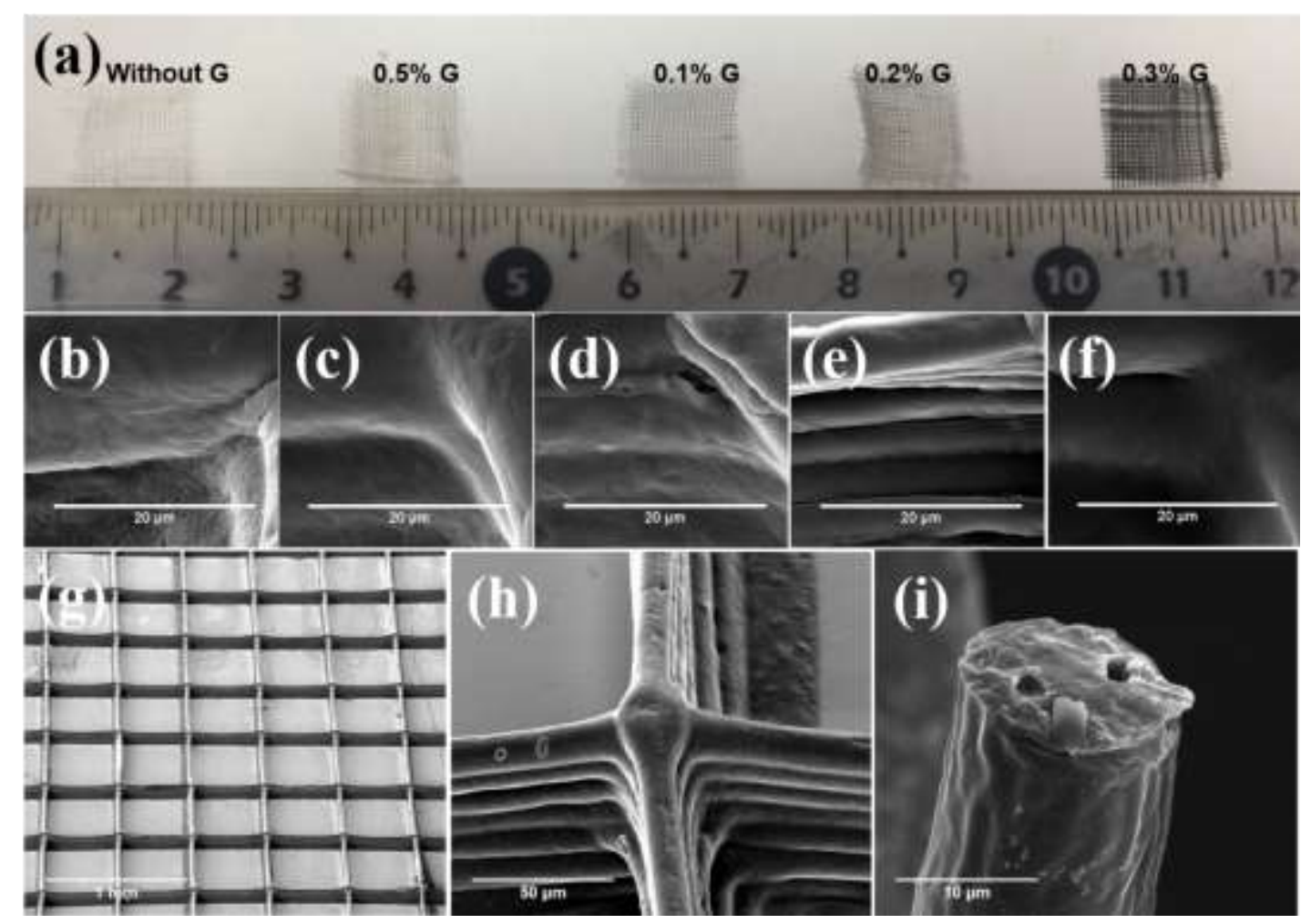

Fig. 4. (a) Pictures of dual-core matrices loading with various concentrations of 
graphene. (b) SEM image of surface of dual-core matrices without graphene. The following images of same magnification are matrices loading (c) $0.05 \%$, (d) $0.1 \%$, (e) $0.2 \%$, and (f) $0.3 \% \mathrm{w} / \mathrm{w}$ graphene. (g) SEM image showing the structures of graphene loaded dual-core matrices. (h) SEM images with high magnification. (i) SEM image of cross-section of dual-core fibers after immersed in DI water. (Fig. 4(g-i) images were all taken with $0.05 \% \mathrm{w} / \mathrm{w}$ graphene loaded matrices)

\subsection{Contact Angle Measurements}

The degree of hydrophobicity of the loaded printed matrices was determined by measuring the direct contact angle between PBS and matrices and is shown in Fig. 5. Five samples with varying graphene concentrations $(0,0.05,0.1,0.2$ and $0.3 \% \mathrm{w} / \mathrm{w})$ were selected to investigate the effect of graphene concentration on the hydrophobicity of dual-core matrices. According to previous studies, the contact angle of pure PCL was over $90^{\circ}$ due to its hydrophobicity [64]. In this study, it was found that the initial contact angle of dual-core matrices without graphene was $112.2^{\circ}$, which is in agreement with previous studies.

However, the hydrophobicity of dual-core matrices decreased with increasing graphene concentration. The initial contact of graphene loaded dual-core matrices with $0.05 \%$, $0.1 \%, 0.2 \%$ and $0.3 \% \mathrm{w} / \mathrm{w}$ was $109^{\circ}, 104^{\circ}, 98^{\circ}$, and $95^{\circ}$, respectively. As shown in Fig. 4(b-f), pure PCL dual-core matrices possessed porous surfaces; introducing a degree of hydrophobicity. In contrast, increasing the graphene concentration from $0.05 \%$ to $0.3 \% \mathrm{w} / \mathrm{w}$ resulted in a smoother surface, which contributes towards hydrophilicity. The contact angle for all matrices after 5 min showed little difference from initial contact angle values, indicating the stability of these matrices with respect to structure. Moreover, the contact angle for all matrices tested were over $90^{\circ}$, proving the inner PEO components had no effect on the hydrophobicity of the matrices and that PEO was completely encapsulated within the PCL fibers.

\subsection{Fourier Transform Infrared (FTIR) and X-ray Diffraction (XRD) Spectroscopy}

Fourier Transform Infrared (FTIR) spectra for graphene loaded dual-core matrices were 
analysed to detect composition and material stability of matrices. The FTIR spectrum of pure materials and graphene-loaded dual-core matrices were displayed in Fig. 5(c) and 5(d), separately. Characteristic absorption bands for PCL could be observed in Fig. $5 \mathrm{c}$, band at $1729 \mathrm{~cm}^{-1}$ belongs to a strong carbonyl $(\mathrm{C}=\mathrm{O})$, and bands between 2868 and $2949 \mathrm{~cm}^{-1}$ were assigned to methylene $\left(\mathrm{CH}_{2}\right)$ groups [57]. Similar bands were observed in spectrums for all dual-core matrices shown in Fig. 5(d). PEO polymer generates an asymmetric stretching peak at $1967 \mathrm{~cm}^{-1}$, while peak at $1473 \mathrm{~cm}^{-1}, 1101 \mathrm{~cm}^{-1}, 961 \mathrm{~cm}^{-1}$ and $840 \mathrm{~cm}^{-1}$ are assigned to $\mathrm{CH}_{2}$ scissoring, $\mathrm{C}-\mathrm{O}-\mathrm{C}$ stretching, $\mathrm{CH}_{2}$ twisting mode and $\mathrm{CH}_{2}$ wagging mode, respectively [65]. Fig. 5(c) also shows that characteristic absorption peaks for DAH mainly appearing at $3340 \mathrm{~cm}^{-1}$ (O-H stretching vibration) and $1616 \mathrm{~cm}^{-1}$ (N-H stretching vibration) [66]. Due to the low quantity of DAH contained in dual-core matrices, only the peak at $3340 \mathrm{~cm}^{-1}$ could be observed for all matrices in Fig. 5(d). In addition, gelatin exhibited its distinguished peaks due to the vibration of carbonyl group $(\mathrm{C}=\mathrm{O})$ at $1650 \mathrm{~cm}^{-1}$ and the stretching vibration of $\mathrm{N}-\mathrm{H}$ at $3291 \mathrm{~cm}^{-1}[67,68]$. For the FTIR spectrum of graphene, there were no significant peaks that were relevant to any functional groups [69], which was also presented in Fig. 5(c). Thus, there was no much difference for the dual-core matrices loading with graphene of various concentrations, as shown in Fig. 5(d).

(a)
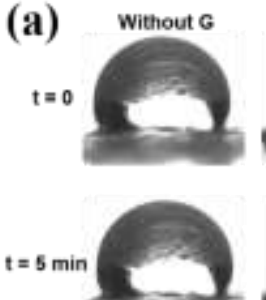

(c)

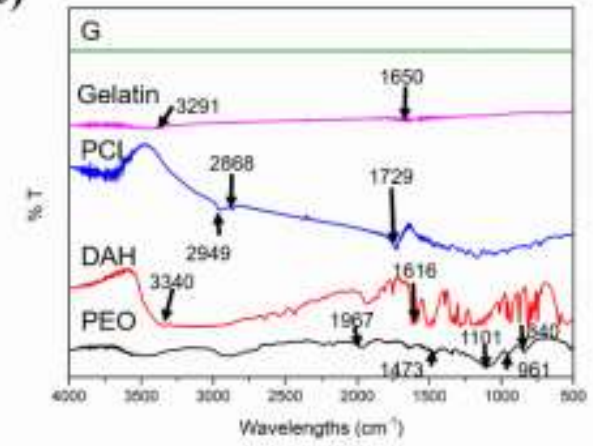

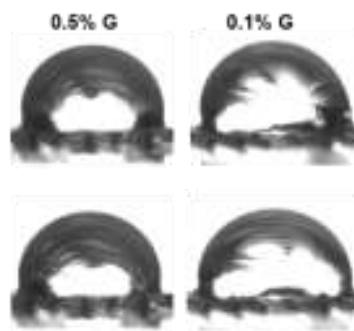

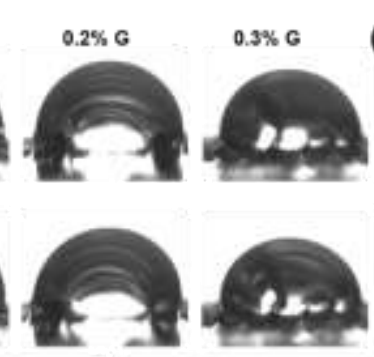

(b)

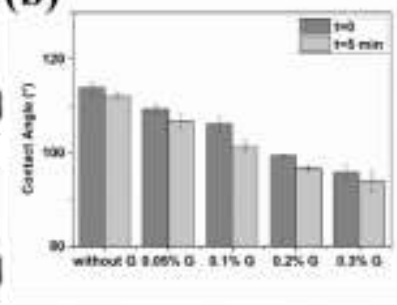

(d)

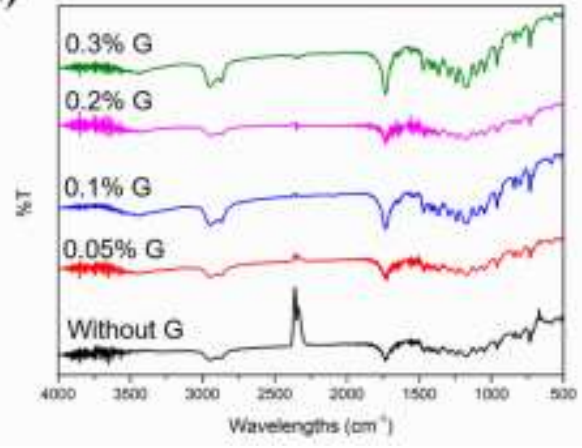


Fig. 5. (a) Real time images and (b) detailed values of contact angles for various concentrations graphene loaded dual-core matrices at initial time and $5 \mathrm{~min}$. (c) FTIR spectrum of pure graphene, gelatin, PCL, DAH and PEO. (d) FTIR spectrum of dualcore matrices loading with different concentrations of graphene.

XRD analysis was carried out to detect any changes in the drug physical form and polymers after the dual-core EHD printing process. Fig. 6(a) shows that pure PCL has two sharp diffraction peaks at $2 \theta=23.5^{\circ}$ and $2 \theta=21.3^{\circ}$, indicating the crystalline nature of PCL [70]. For pure PEO, two peaks were observed around $2 \theta=19.1^{\circ}$ and $2 \theta$ $=23.2^{\circ}[71]$. However, only the peak at $2 \theta=19.1^{\circ}$ is apparent for dual-core matrices, suggesting peaks had overlapped. These results also indicate PEO crystallinity is lower in the fabricated matrices when compared to the pristine material. In Fig. 6(a), a broad peak is evident at $26^{\circ}$ for pure graphene indicating crystallinity [72] which masked the sharp peaks for PEO and PCL. (Fig. 6(b). Moreover, peaks indicative of crystallinity for pure DAH and gelatin disappeared in the matrices, suggesting that DAH and gelatin were amorphous or dispersed in the matrices following EHD processing.

\subsection{Mechanical Testing}

Mechanical properties of printed matrices can be modified by the inclusion of graphene. Fig. 6(c) shows the stress-strain curves of graphene-loaded matrices. The matrices without graphene demonstrated the greatest tensile strength $(14.3 \mathrm{MPa})$. When increasing graphene concentration from $0.05 \%$ to $0.3 \% \mathrm{w} / \mathrm{w}$, the tensile strength of the matrices decreased from 5.5 $\mathrm{MPa}$ to $1.1 \mathrm{MPa}$. In addition, elongation at break also decreased when the graphene was incorporated. This was due to the reason that addition of graphene increased brittleness and reduced elasticity of the dual-core matrices. The elastic modulus of these matrices is presented in Fig. 6(d). It was found that elastic modulus decreased with increasing graphene concentration, which also explains the stress-strain curves. According to previous studies, pure graphene exhibits exceptionally high elastic modulus (1 TPa) and intrinsic strength (130 GPa) [73], and for these reasons graphene is an attractive filler material for the preparation of 
533 functional polymer composites. The mechanical strength of such composites is 534 dependent on the shape and degree of interfacial bonding between the polymer and 535 filler material [74]. Furthermore, mechanical and tribological properties vary and depend on the percentage (composition) of filler compared to homogeneous and pristine materials [75]. Extensive studies on nanoscale filler-polymer composite materials have shown improved mechanical properties with increasing filler content. The vast majority of these relate to film (sheet) or dense structures with limited voids (micron scales pores between struts). Such properties have also been shown for composite scaffolds, again where the strut size (between pores) are coarse (e.g. for tissue engineering applications using nanoscale HA and polymer) [76]. However, using filler alongside micron and sub-micron scaled fibers does not yield similar mechanical properties due to greater porosity and reduced composite strut/fibre diameter. Unlike films, non-porous or slightly porous composites, fibrous composites are weaker in their mechanical properties and this can be seen clearly when comparing such structures made from similar components. In this regard the addition of filler to sub-micron and micron scaled diners is likely to impact mechanical properties. This is further compounded by the alignment and core shell structures in this study. Hence, increasing the graphene concentration from $0.05 \%$ to $0.3 \% \mathrm{w} / \mathrm{w}$ results in lower mechanical properties (of dualcore matrices). At this scale and porosity; polymer chains are discontinuous and chain entanglement and interaction is reduced due to the presence of small quantities of graphene and greater void space. 

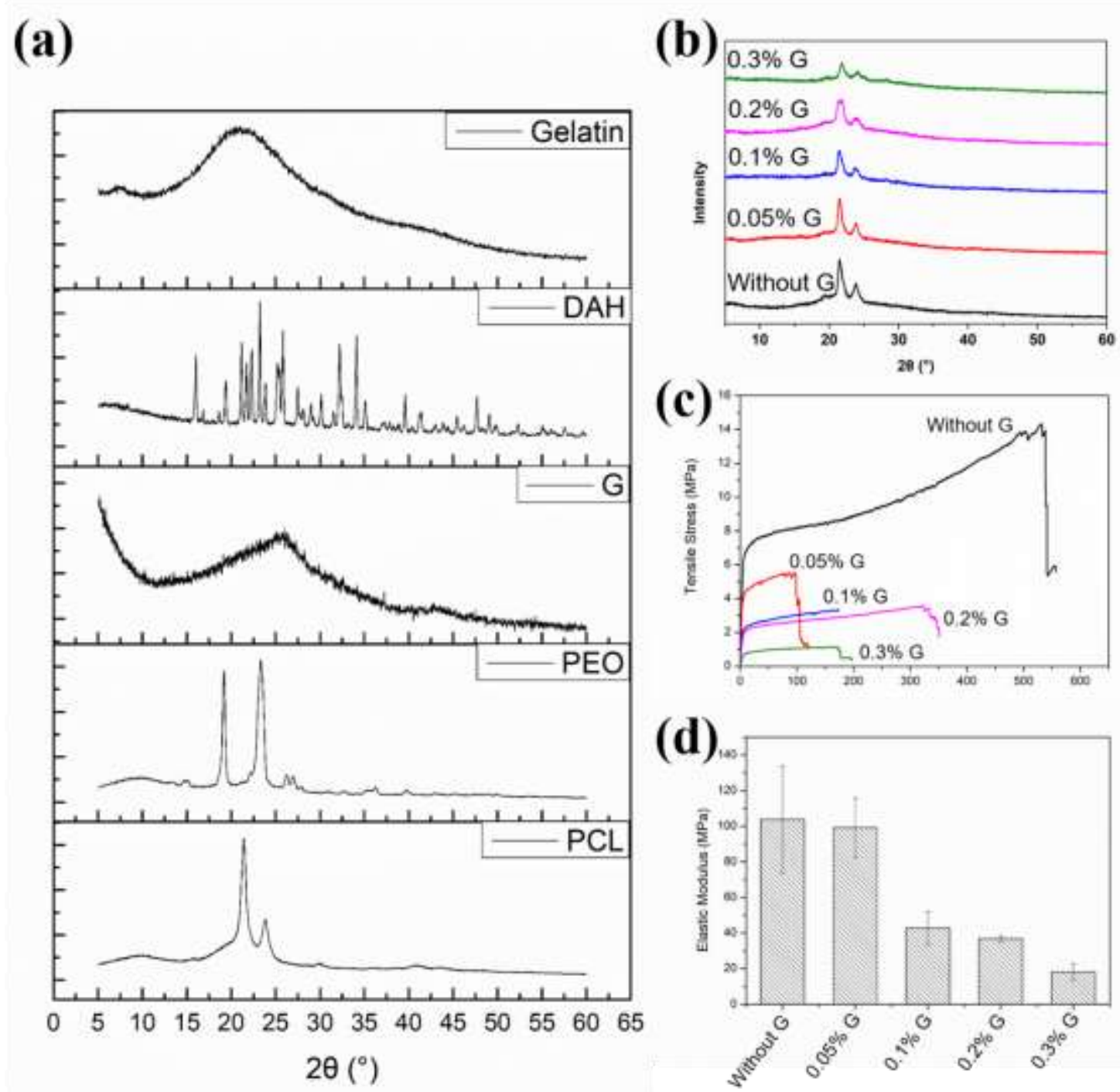

Fig. 6. (a) XRD patterns of pure DAH, graphene, gelatin, PEO and PCL. (b) XRD patterns of graphene loaded dual-core matrices. (c) Stress-strain curves and (d) elastic modulus for graphene loaded dual-core matrices.

\subsection{Drug Release from Graphene loaded Dual-core Matrices}

562 Fig. 7 shows the cumulative release profiles of DAH from printed dual-core matrices.

563 For all samples, the release profiles were biphasic. The release process started with a 564 rapid initial release period (up to $30 \mathrm{~min}$ ) and was followed by a slow release stage 565 (from 30 to $600 \mathrm{~min}$ ). As DAH was incorporated in PEO components and PEO is 566 soluble in PBS, DAH is almost completely released at $600 \mathrm{~min}$. Higuchi model and 567 Korsmeyer-Peppas models were applied to validate drug release mechanisms of DAH 568 from the dual-core matrices. As shown in Table 1, the $n$ values of the Korsmeyer-Peppas 
574

model were $0.1737,0.1564,0.1709,0.1085$ and 0.1353 for matrices without graphene, and matrices loaded with $0.05,0.1,0.2,0.3 \% \mathrm{w} / \mathrm{w}$ graphene, respectively, indicating release of DAH was primarily Fickian Diffusion based. The Higuchi model collated high correlation coefficients; mimicking the data shown with the Korsmeyer Peppas model; showing DAH release was certainly controlled by diffusion.

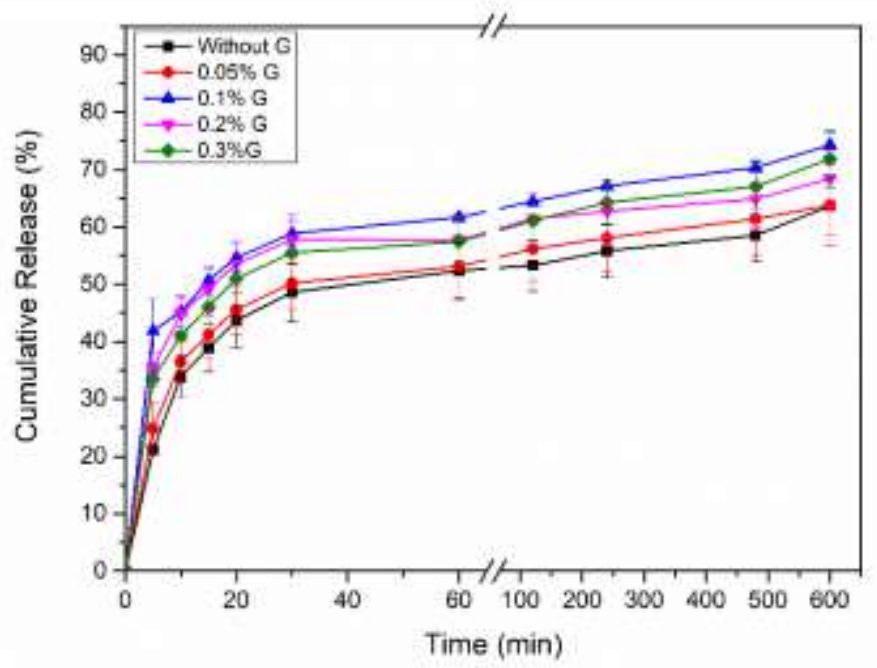

Fig. 7. DAH release behaviors from various concentrations of graphene loaded dualcore matrices.

Table 1. Fitting parameters of DAH release from various concentrations of graphene loaded dual-core matrices.

\begin{tabular}{llll}
\hline Sample & Higuchi $\left(\mathbf{R}^{\mathbf{2}}\right)$ & Korsmeyer-Peppas $\left(\mathbf{R}^{\mathbf{2}}\right)$ & $\mathbf{n}$ \\
\hline Without G & 0.9504 & 0.7766 & 0.1737 \\
\hline $\mathbf{0 . 0 5 \%}$ G & 0.9546 & 0.8125 & 0.1564 \\
\hline $\mathbf{0 . 1 \%}$ G & 0.9841 & 0.9541 & 0.1709 \\
\hline $\mathbf{0 . 2 \%}$ G & 0.9694 & 0.8828 & 0.1085 \\
\hline $\mathbf{0 . 3 \%}$ G & 0.9866 & 0.8938 & 0.1353
\end{tabular}

\subsection{PC12 Cell Morphology Study and CCK-8 Cell Viability test}

Cell morphology is an important factor in determining material biocompatibility. Table 2 details each sample used for in vitro cell tests. As graphene-loaded dual-core matrices 
have great potential in nerve restoration, an ideal environment for cell attachment and proliferation is essential. Fluorescent images of PC12 cells cultured on various dualcore matrices for 3 days are presented in Fig. 8. It could be observed in Fig. 8(a-a1) that, PC12 cells cultured for the control group only showed round and two-dimensional morphology, with little to none cell extension. However, for cells cultured on graphene loaded dual-core matrices, their morphology was three-dimensional, and cell spreading and neurite outgrowth is apparent. In addition, the number of cells attached on the matrices without graphene was significantly lower than cells on graphene loaded matrices (Fig. 8(b-f)). These results show that increasing the graphene concentration in matrices enhances biocompatibility and improves cell extension. Cell viability indicates the potential toxic risks of dual-core matrices, which is displayed as a percentage of viable cells within the total cell population. CCK-8 tests were used to assess biocompatibility with particular emphasis on graphene. According to previous studies, cell viability tests have been performed at various time points post incubation e.g. for 8 hours[77], 3 days [78] and for more prolonged periods (14 days)[79]. Cell viability indicates potential toxicity of graphene loaded matrices, expressed as a percentage of viable cells within the total cell population, and calculated by comparing the test group (dual-core matrices) to the control (no sample). In this study, the cells had spread and covered engineered matrices after 3 days. Furthermore, based on DAH release data, the inner PEO material has dissolved completely at this stage. Therefore, from our perspective, testing graphene based matrices at 3 days is sufficient for biocompatibility evaluation. As shown in Fig. 9, cell viability for dual-core matrices loading with $0.05 \%$, $0.1 \%, 0.2 \%$ and $0.3 \%$ graphene were $92.9 \%, 96.2 \%, 98.1 \%$ and $98.7 \%$ compared to the control group (100\%), separately. While cell viability for matrices without graphene, and matrices containing $(0.05 \% \mathrm{w} / \mathrm{w})$ graphene but without gelatin and DAH were $37.3 \%$ and $80.2 \%$, respectively.

The results indicate that graphene loaded dual-core matrices show good biocompatibility, and increasing graphene concentration enhances cell viability. In addition, incorporation of gelatin and DAH also has a positive effect on cell viability. 
Previous studies involving graphene based materials have exhibited both biocompatibility and toxicity towards cells. Interactions between graphene-loaded materials and membrane lipids are known to cause damage and be cytotoxic. Furthermore, small size and sharp edge definitions of graphene-loaded materials enable these materials to disrupt cellular membranes causing leakage of cytoplasmic contents [73].

In this study, graphene was loaded in dual-core fiber matrices. The overall size of engineered matrices was too coarse to penetrate into PC12 cells. The concentration of graphene in the PCL shell was varied $(0,0.05,0.1,0.2$ and $0.3 \% \mathrm{w} / \mathrm{w})$ to investigate its loading concentration on cell viability and migration rate. As shown in Fig. 8 (b-f), PC12 cells were observed on all matrices (i.e. constructs hosting graphene at all concentrations). Previous studies have used CCK-8 tests to show material biocompatibility [80]. Cell viability on graphene-loaded matrices and control group (TCP culture dish) were near identical, indicating good biocompatibility of the composite matrices. In contrast, for dual-core matrices without graphene, cell viability was less than $40 \%$. And for the dual-core matrices with $(0.05 \% \mathrm{w} / \mathrm{w})$ and without DAH as well as gelatin, the cell viability was $\sim 80.5 \%$, which was also lower than the dualcore matrices with identical graphene concentration for both DAH and gelatin. Therefore, DAH and gelatin incorporation into matrices improves cell biocompatibility. Based on these results, addition of graphene, gelatin and DAH in dual-core matrices enhances their biocompatibility. Furthermore, cell migration (and adhesion) has been evaluated through focal adhesions [73]. According to previous studies, cells (L929) cultured on graphene films are known to illicit stronger vinculin expression through the quantification of focal adhesions [81]. Research related to the effect of gelatin and DAH on cell migration is well documented $[82,83]$, and for this reason was not explored in this in this study.

Table 2. In vitro cell culture results. (The concentration of graphene is in relation to PCL weight). Concentrations of gelatin and DAH are in relation to PEO weight. "- 
645 "represents non-inclusion)

646

\begin{tabular}{cccc}
\hline Cell Test Sample & Graphene \% & Gelatin\% & DAH\% \\
\hline Control 1 (TCPs) & - & - & - \\
\hline Control 2 & 0.05 & - & - \\
\hline T1 & 0 & 50 & 5 \\
\hline T2 & 0.05 & 50 & 5 \\
\hline T3 & 0.1 & 50 & 5 \\
\hline T4 & 0.2 & 50 & 5 \\
\hline T5 & 0.3 & 50 & \\
\hline
\end{tabular}

647

648 


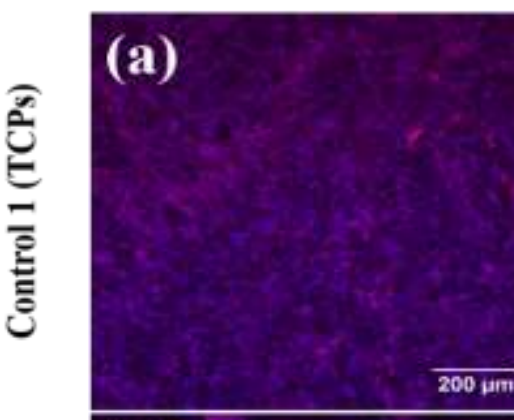

(a1)

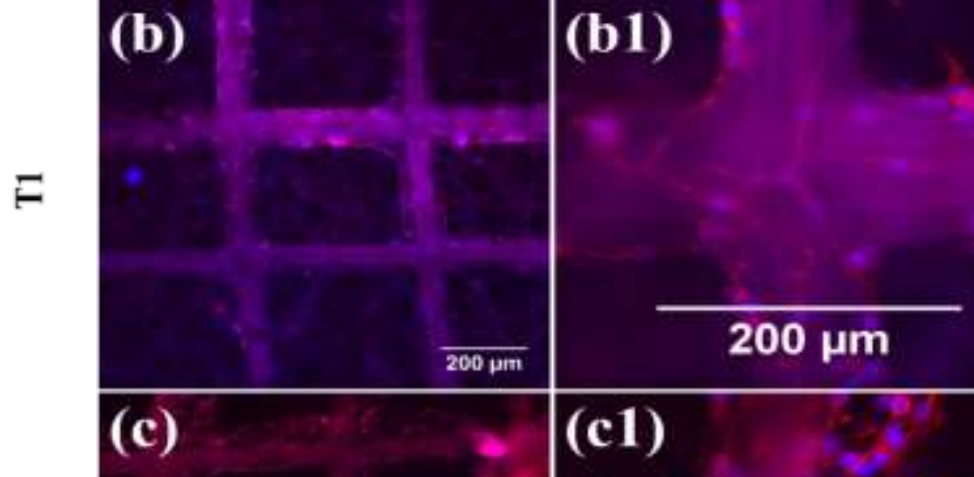

I

2 (d1)

(d) $200 \mu \mathrm{m}$

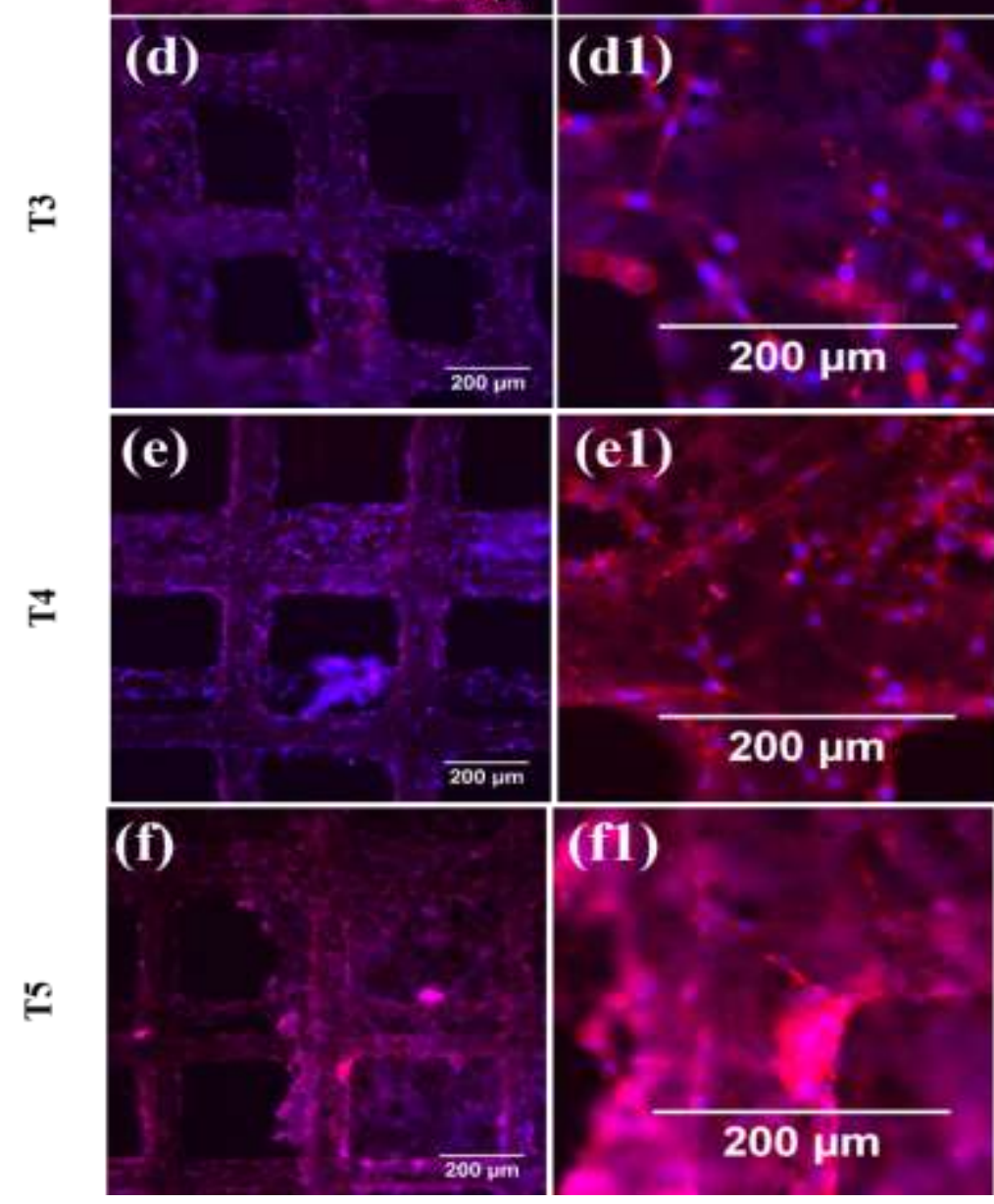


654

Fig. 8. Fluorescence micrographs of PC12 cells grown on (a) control group (only TCPs), multi-cores matrices (b) without graphene, and matrices loading with (c) $0.05 \% \mathrm{w} / \mathrm{w}$, (d) $0.1 \% \mathrm{w} / \mathrm{w}$, (e) $0.2 \% \mathrm{w} / \mathrm{w}$ and (f) $0.3 \% \mathrm{w} / \mathrm{w}$ concentration of graphene. (a1)- (f1) are the images with higher magnification.

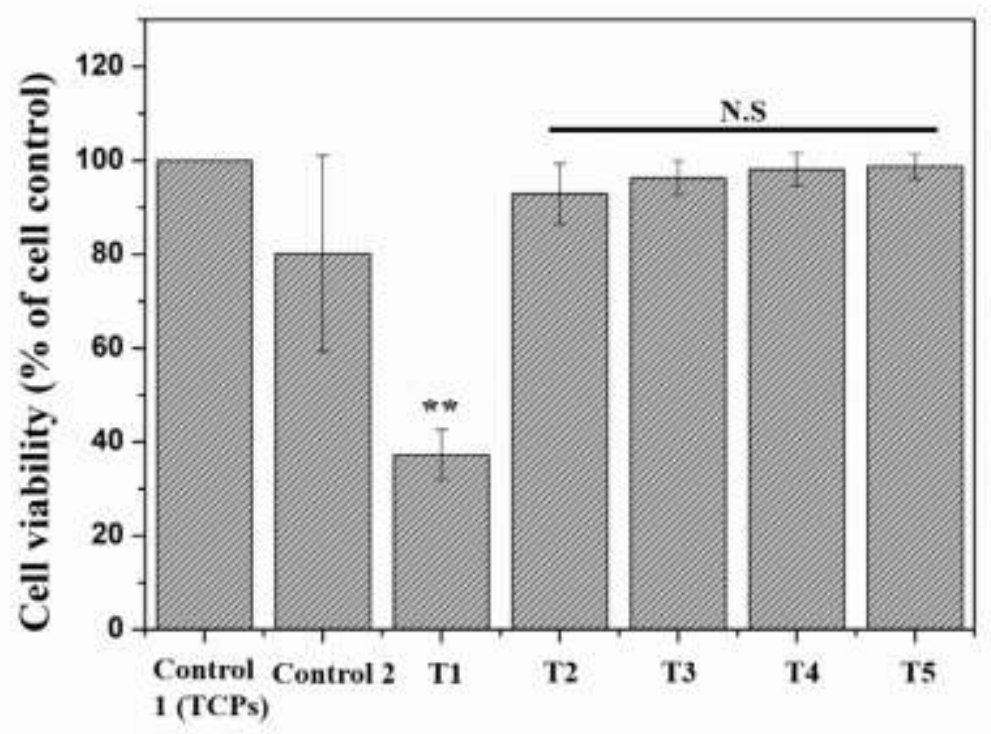

Fig. 9. CCK-8 test on graphene loaded dual-core matrices after 3 days culture. And the control was the cells cultured just on the $96-$ well plate. (** represents significant difference, $p<0.01$; N.S. represents there was no significant difference.)

\subsection{PC12 Cell Migration Assay}

The effect of graphene concentration on PC12 cell migration was investigated. Fig. 10 presents cell migration speed of scratched PC12 cell monolayer in the presence of both control groups, matrices containing $0.05 \%$ graphene without gelatin and $\mathrm{DAH}$, and dual-core matrices loaded with various graphene concentrations. Assessments were made at 24 and $72 \mathrm{~h}$ of incubation. Migration percentage was measured via ImageJ software and is shown in Fig. 11. The results show that the monolayer appears irregular after an initial $24 \mathrm{~h}$ of incubation (Fig. 10(a1-g1)). Cell polarization is apparent with protrusions found in all samples. Dual-core matrices without graphene exhibit slowest cell migration speed $(\sim 16 \%)$. During the first $24 \mathrm{~h}$, the migration rate is shown to increase with increasing graphene concentration (within matrices). For the matrices 
670 without gelatin and $\mathrm{DAH}$, the migration rate was almost identical to matrices loaded 671 with graphene (Fig. 11), indicating that graphene plays the main role in PC12 cell 672 migration rate. After incubation for $72 \mathrm{~h}$, cells cultured on matrices without graphene 673 were apoptotic (Fig. 10(c2)), which is in accordance with results of CCK-8 test. For 674 other samples, cell migration to sparse areas was observed leading to a reduction in cell 675 density in the border area (Fig. 10(a2-b2) and (d2-g2)). Cells cultured on the dual-core 676 matrices loaded with $0.05 \%$ graphene displayed the highest migration rate $(>80 \%)$, 677 suggesting that a moderate quantity of graphene in matrices is sufficient to enhance cell 678 migration. Cell migration tests show that graphene loaded dual-core matrices have great 679 potential to promote nerve restoration. 


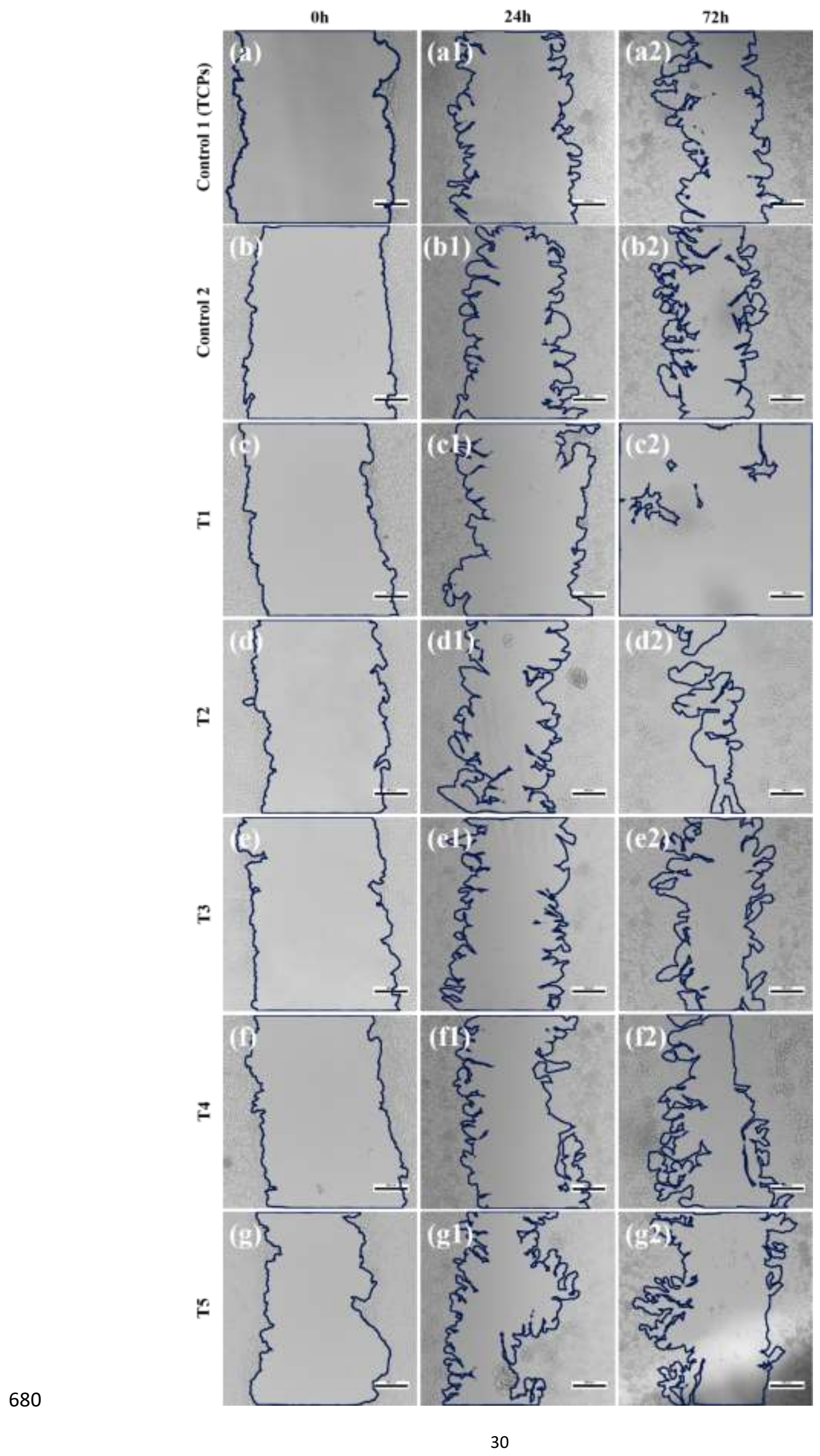


Fig. 10. Micrographs of PC12 cells migrating into a scratch area over a $72 \mathrm{~h}$ period in (a) the absence (control), (b) samples containing $0.05 \%$ graphene without gelatin and DAH, and dual-core matrices loaded with (c) $0 \%$, (d) $0.05 \%$, (e) $0.1 \%$ and (f) $0.2 \%$ $\mathrm{w} / \mathrm{w}$ graphene. The blue lines in these images showed the edges of migration area. The scale bar is $200 \mu \mathrm{m}$.

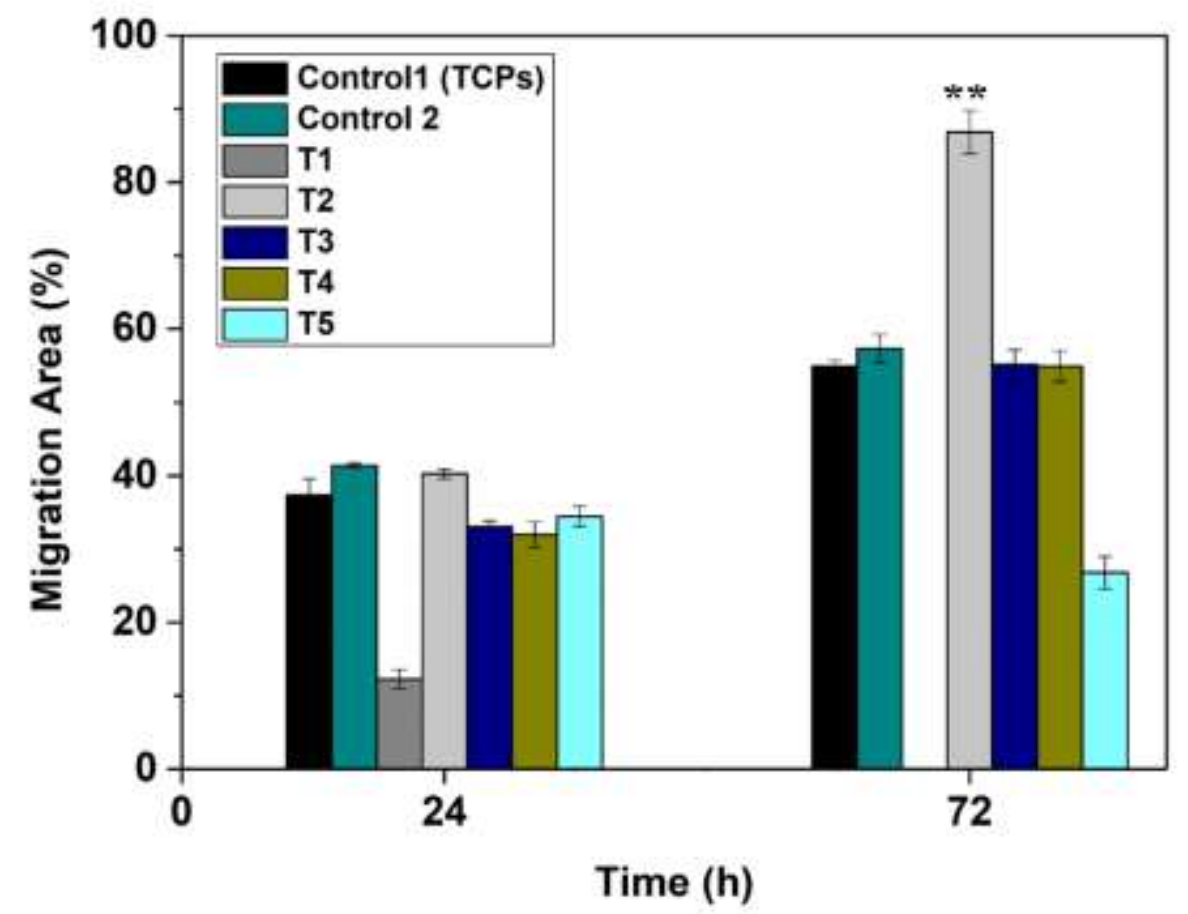

Fig. 11. Evaluation of migration rate by the curves of the percentage of migration area as a function of incubation time. (** represents significant difference, $p<0.01$ )

\section{Conclusion}

Novel graphene-loaded polycaprolactone (PCL)/polyethylene oxide (PEO) dual-core matrices were fabricated via an EHD printing method. For dual-core matrices, graphene was loaded into the outer PCL layer, while gelatin and dopamine hydrochloride (DAH) were encapsulated in two separate internal PEO cores to improve biocompatibility. The optimum PEO solution concentration was $5 \% \mathrm{w} / \mathrm{w}$ for dual-core fiber fabrication. This study also investigated the influence of process parameters (applied voltage, inner flow rate, outer flow rate and collecting $X-Y-Z$ stage speed) on the morphology of dual-core fibers. It was found that graphene loaded dual-core matrices had two internal cores and 
graphene content correlated with a smoother non-porous surface. Increasing the graphene content reduced matrix elasticity and dual-core matrices loaded with $0.3 \%$ graphene exhibited lowest elastic modulus. DAH release behavior from matrices with various graphene concentrations were all diffusion-controlled and showed no significant difference, with complete release of DAH after 600 min. Graphene loaded dual-core matrices show good biocompatibility, and gelatin (as well DAH) compositions also improve PC12 cell attachment. Matrices loaded with $0.05 \% \mathrm{w} / \mathrm{w}$ graphene promote maximal cell migration. In summary, dual-core matrices show clear potential as nerve graft materials.

\section{Acknowledgements}

This research was financially supported by the National Nature Science Foundation of China (No. 81771960), the Fundamental Research Funds for the Central Universities (2017QNA5017) and Key Technologies R\&D Program of Zhejiang Province (2015C02035).

\section{Reference}

[1] N. Golafshan, M. Kharaziha, and M. Fathi, Tough and conductive hybrid graphene-PVA: Alginate fibrous scaffolds for engineering neural construct, Carbon. 111 (2017)752-763.

[2] S. Shrestha, B.K. Shrestha, J.I. Kim, S.W. Ko, C.H. Park, and C.S. Kim, Electrodeless coating polypyrrole on chitosan grafted polyurethane with functionalized multiwall carbon nanotubes electrospun scaffold for nerve tissue engineering, Carbon. 136 (2018)430-443.

[3] P. Gupta, S. Sharan, P. Roy, and D. Lahiri, Aligned carbon nanotube reinforced polymeric scaffolds with electrical cues for neural tissue regeneration, Carbon. 95 (2015)715-724.

[4] J.R. Kim, S.H. Oh, G.B. Kwon, U. Namgung, K.S. Song, B.H. Jeon, and J.H. Lee, Acceleration of Peripheral Nerve Regeneration through Asymmetrically Porous Nerve Guide Conduit Applied with Biological/Physical Stimulation, Tissue.Eng. Part. A. 19 (2013)2674-2685.

[5] D.J. Lee, A. Fontaine, X.Z. Meng, and D. Park, Biomimetic Nerve Guidance Conduit Containing Intraluminal Microchannels with Aligned Nanofibers Markedly Facilitates in Nerve Regeneration, Acs. Biomater. Sci. Eng. 2 (2016) 1403-1410.

[6] B.K. Lee, Y.M. Ju, J.G. Cho, J.D. Jackson, S.J. Lee, A. Atala, and J.J. Yoo, End- 
to-side neurorrhaphy using an electrospun PCL/collagen nerve conduit for complex peripheral motor nerve regeneration, Biomaterials. 33 (2012)90279036.

[7] A.J. Reid, A.C. de Luca, A. Faroni, S. Downes, M. Sun, G. Terenghi, and P.J. Kingham, Long term peripheral nerve regeneration using a novel PCL nerve conduit, Neurosci. Lett. 544 (2013)125-130.

[8] M. Sun, P.J. Kingham, A.J. Reid, S.J. Armstrong, G. Terenghi, and S. Downes, In vitro and in vivo testing of novel ultrathin PCL and PCL/PLA blend films as peripheral nerve conduit, J. Biomed. Mater. Res. A. 93A (2010)1470-1481.

[9] J.Q. Liu, L. Cui, and D. Losic, Graphene and graphene oxide as new nanocarriers for drug delivery applications, Acta. Biomater. 9 (2013)9243-9257.

[10] L.Z. Feng and Z.A. Liu, Graphene in biomedicine: opportunities and challenges, Nanomedicine. 6 (2011)317-324.

[11] L.Y. Feng, L. Wu, and X.G. Qu, New Horizons for Diagnostics and Therapeutic Applications of Graphene and Graphene Oxide, Adv.Mater. 25 (2013)168-186.

[12] Z. Liu, J.T. Robinson, S.M. Tabakman, K. Yang, and H.J. Dai, Carbon materials for drug delivery \& cancer therapy, Mater. Today. 14 (2011)316-323.

[13] Y. Qian, X. Zhao, Q. Han, W. Chen, H. Li, and W. Yuan, An integrated multilayer 3D-fabrication of PDA/RGD coated graphene loaded PCL nanoscaffold for peripheral nerve restoration, Nat. Commun. 9 (2018)323.

[14] N. Li, Q. Zhang, S. Gao, Q. Song, R. Huang, L. Wang, L.W. Liu, J.W. Dai, M.L. Tang, and G.S. Cheng, Three-dimensional graphene foam as a biocompatible and conductive scaffold for neural stem cells, Sci. Rep. 3 (2013)6.

[15] Q. Ma, L. Yang, Z. Jiang, Q. Song, M. Xiao, D. Zhang, X. Ma, T. Wen, and G. Cheng, Three-Dimensional Stiff Graphene Scaffold on Neural Stem Cells Behavior, Acs. Appl. Mater. Inter. 8 (2016)34227-34233.

[16] H.T. Liu, Y.Q. Liu, and D.B. Zhu, Chemical doping of graphene, J. Mater. Chem. 21 (2011)3335-3345.

[17] Y. Gu, J.B. Zhu, C.B. Xue, Z.M.Y. Li, F. Ding, Y.M. Yang, and X.S. Gu, Chitosan/silk fibroin-based, Schwann cell-derived extracellular matrixmodified scaffolds for bridging rat sciatic nerve gaps, Biomaterials. 35 (2014) 2253-2263.

[18] R. Ravichandran, M. Griffith, and J. Phopase, Applications of self-assembling peptide scaffolds in regenerative medicine: the way to the clinic, J. Mater. Chem. B. 2 (2014)8466-8478.

[19] R.J. Wade and J.A. Burdick, Advances in nanofibrous scaffolds for biomedical applications: From electrospinning to self-assembly, Nano. Today. 9 (2014)722742.

[20] S. Tang, J. Zhu, Y. Xu, A.P. Xiang, M.H. Jiang, and D. Quan, The effects of gradients of nerve growth factor immobilized PCLA scaffolds on neurite outgrowth in vitro and peripheral nerve regeneration in rats, Biomaterials. 34 (2013)7086-7096.

[21] J. Xie, M.R. MacEwan, W. Liu, N. Jesuraj, X. Li, D. Hunter, and Y. Xia, Nerve Guidance Conduits Based on Double-Layered Scaffolds of Electrospun 
[22] A.R. Dixon, S.H. Jariwala, Z. Bilis, J.R. Loverde, P.F. Pasquina, and L.M.

Nanofibers for Repairing the Peripheral Nervous System, Acs. Appl. Mater. Inter. 6 (2014)9472-9480. Alvarez, Bridging the gap in peripheral nerve repair with $3 \mathrm{D}$ printed and bioprinted conduits, Biomaterials. 186 (2018)44-63.

[23] B. Wang, S. Wu, Z. Ahmad, J.-S. Li, and M.-W. Chang, Co-printing of vertical axis aligned micron-scaled filaments via simultaneous dual needle electrohydrodynamic printing, Eur. Polym. J. 104 (2018)81-89.

[24] K.J. Lee, T.-H. Park, S. Hwang, J. Yoon, and J. Lahann, Janus-Core and Shell Microfibers, Langmuir. 29 (2013)6181-6186.

[25] J.S.M. Zanjani, B.S. Okan, I. Letofsky-Papst, M. Yildiz, and Y.Z. Menceloglu, Rational design and direct fabrication of multi-walled hollow electrospun fibers with controllable structure and surface properties, Eur. Polym. J. 62 (2015)6676.

[26] M.B. Gebeyehu, Y.H. Chang, A.K. Abay, S.Y. Chang, J.Y. Lee, C.M. Wu, T.C. Chiang, and R.I. Murakami, Fabrication and characterization of continuous silver nanofiber/polyvinylpyrrolidone (AgNF/PVP) core-shell nanofibers using the coaxial electrospinning process, Rsc. Adv. 6 (2016)54162-54168.

[27] H.W. Mao, P.B. Ma, and G.M. Jiang, Filtration Property of Monofilament CoreShell Mesh Fabric Treated via Tourmaline Hot Coating, Autex. Res. J. 19 (2019) 127-133.

[28] X.Y. Wang, Y.H. Yuan, X.C. Huang, and T.L. Yue, Controlled release of protein from core-shell nanofibers prepared by emulsion electrospinning based on green chemical, J. Appl. Polym. Sci. 132 (2015)9.

[29] P. Coimbra, P. Santos, P. Alves, S.P. Miguel, M.P. Carvalho, K.D. de Sa, I.J. Correia, and P. Ferreira, Coaxial electrospun PCL/Gelatin-MA fibers as scaffolds for vascular tissue engineering, Colloid. Surf. B. 159 (2017)7-15.

[30] L.E. Sperling, K.P. Reis, P. Pranke, and J.H. Wendorff, Advantages and challenges offered by biofunctional core-shell fiber systems for tissue engineering and drug delivery, Drug. Discov. Today. 21 (2016)1243-1256.

[31] C.-Y. Wang, J.-J. Liu, C.-Y. Fan, X.-M. Mo, H.-J. Ruan, and F.-F. Li, The Effect of Aligned Core-Shell Nanofibres Delivering NGF on the Promotion of Sciatic Nerve Regeneration, J. Biomat. Sci-Polym. E. 23 (2012)167-184.

[32] K. Nischala, T.N. Rao, and N. Hebalkar, Silica-silver core-shell particles for antibacterial textile application, Colloid. Surf. B. 82 (2011)203-208.

[33] A. Sarvi and U. Sundararaj, Electrical Permittivity and Electrical Conductivity of Multiwall Carbon Nanotube-Polyaniline (MWCNT-PANi) Core-Shell Nanofibers and MWCNT-PANi/polystyrene Composites, Macromol. Mater. Eng. 299 (2014)1013-1020.

[34] Z.Q. Xiong, Y. Zhang, X.Y. Du, P.A. Song, and Z.P. Fang, Green and Scalable Fabrication of Core-Shell Biobased Flame Retardants for Reducing Flammability of Polylactic Acid, Acs. Sustain. Chem. Eng. 7 (2019)8954-8963.

[35] A. Subramanian, U.M. Krishnan, and S. Sethuraman, Fabrication of uniaxially aligned 3D electrospun scaffolds for neural regeneration, Biomed. Mater. 6 
(2011)10.

[36] A.N. Yang, Z.B. Huang, G.F. Yin, and X.M. Pu, Fabrication of aligned, porous and conductive fibers and their effects on cell adhesion and guidance, Colloid. Surf. B. 134 (2015)469-474.

[37] J.Y. Feng, D.T. Zhang, M.F. Zhu, and C.Y. Gao, Poly(L-lactide) melt spun fiberaligned scaffolds coated with collagen or chitosan for guiding the directional migration of osteoblasts in vitro, J. Mater. Chem. B. 5 (2017)5176-5188.

[38] S.K. Vimal, N. Ahamad, and D.S. Katti, A simple method for fabrication of electrospun fibers with controlled degree of alignment having potential for nerve regeneration applications, Mat. Sci. Eng. C-Mater. 63 (2016)616-627.

[39] K. Sheets, S. Wunsch, C. Ng, and A.S. Nain, Shape-dependent cell migration and focal adhesion organization on suspended and aligned nanofiber scaffolds, Acta. Biomater. 9 (2013)7169-7177.

[40] S.Y. Chew, R. Mi, A. Hoke, and K.W. Leong, Aligned protein-polymer composite fibers enhance nerve regeneration: A potential tissue-engineering platform, Adv. Funct. Mater. 17 (2007)1288-1296.

[41] H.B. Wang, M.E. Mullins, J.M. Cregg, A. Hurtado, M. Oudega, M.T. Trombley, and R.J. Gilbert, Creation of highly aligned electrospun poly-L-lactic acid fibers for nerve regeneration applications, J. Nerural. Eng. 6 (2009)016001.

[42] M.M.L. Arras, C. Grasl, H. Bergmeister, and H. Schima, Electrospinning of aligned fibers with adjustable orientation using auxiliary electrodes, Sci. Technol.Adv. Mat. 13 (2012)035008.

[43] J.K. He, F.Y. Xu, R.N. Dong, B.L. Guo, and D.C. Li, Electrohydrodynamic 3D printing of microscale poly (epsilon-caprolactone) scaffolds with multi-walled carbon nanotubes, Biofabrication. 9 (2017)10.

[44] S. Vijayavenkataraman, S. Thaharah, S. Zhang, W.F. Lu, and J.Y.H. Fuh, 3DPrinted PCL/rGO Conductive Scaffolds for Peripheral Nerve Injury Repair, Artif. Organs. 43 (2019)515-523.

[45] R.H. Zhou and H.J. Gao, Cytotoxicity of graphene: recent advances and future perspective, Wires. Nanomed. Nanobi. 6 (2014)452-474.

[46] Y. Zhu, J.C. Zhang, Y.M. Zheng, Z.B. Huang, L. Feng, and L. Jiang, Stable, superhydrophobic, and conductive polyaniline/polystyrene films for corrosive enviromnents, Adv. Funct. Mater. 16 (2006)568-574.

[47] J. Greeley, T.F. Jaramillo, J. Bonde, I.B. Chorkendorff, and J.K. Norskov, Computational high-throughput screening of electrocatalytic materials for hydrogen evolution, Nat. Mater. 5 (2006)909-913.

[48] J. Njagi, M.M. Chernov, J.C. Leiter, and S. Andreescu, Amperometric Detection of Dopamine in Vivo with an Enzyme Based Carbon Fiber Microbiosensor, Anal. Chem. 82 (2010)989-996.

[49] Y. Fu, P. Li, Q. Xie, X. Xu, L. Lei, C. Chen, C. Zou, W. Deng, and S. Yao, OnePot Preparation of Polymer-Enzyme-Metallic Nanoparticle Composite Films for High-Performance Biosensing of Glucose and Galactose, Adv. Funct. Mater. 19 (2009)1784-1791.

[50] M.S. Khan, S. Pandey, T. Abou, M.L. Bhaisare, and H.F. Wu, Controlled 
delivery of dopamine hydrochloride using surface modified carbon dots for neuro diseases, Colloid. Surf. B. 134 (2015)140-146.

[51] F. Ghorbani, A. Zamanian, and A. Aidun, Bioinspired polydopamine coatingassisted electrospun polyurethane-graphene oxide nanofibers for bone tissue engineering application, J. Appl. Polym. Sci. 136 (2019)9.

[52] W.C. Low, P.O. Rujitanaroj, D.K. Lee, P.B. Messersmith, L.W. Stanton, E. Goh, and S.Y. Chew, Nanofibrous scaffold-mediated REST knockdown to enhance neuronal differentiation of stem cells, Biomaterials. 34 (2013)3581-3590.

[53] R. Liu, H. Zhang, F. Zhang, X. Wang, X. Liu, and Y. Zhang, Polydopamine doped reduced graphene oxide/mesoporous silica nanosheets for chemophotothermal and enhanced photothermal therapy, Mat. Sci. Eng. C-Mater. 96 (2019)138-145.

[54] D.W. Li, L. Luo, Z.Y. Pang, L. Ding, Q.Q. Wang, H.Z. Ke, F.L. Huang, and Q.F. Wei, Novel Phenolic Biosensor Based on a Magnetic Polydopamine-LaccaseNickel Nanoparticle Loaded Carbon Nanofiber Composite, Acs. Appl. Mater. Inter. 6 (2014)5144-5151.

[55] Z.X. Meng, Y.S. Wang, C. Ma, W. Zheng, L. Li, and Y.F. Zheng, Electrospinning of PLGA/gelatin randomly-oriented and aligned nanofibers as potential scaffold in tissue engineering, Mat. Sci. Eng. C-Mater. 30 (2010)1204-1210.

[56] S. Vijayavenkataraman, S. Zhang, S. Thaharah, G. Sriram, W.F. Lu, and J.Y.H. Fuh, Electrohydrodynamic Jet 3D Printed Nerve Guide Conduits (NGCs) for Peripheral Nerve Injury Repair, Polymers. 10 (2018)25.

[57] B. Wang, H. Zheng, M. Chang, Z. Ahmad, and J. Li, Hollow polycaprolactone composite fibers for controlled magnetic responsive antifungal drug release, Colloid. Surf. B. 145 (2016)757-767.

[58] Y. Gao, M.W. Chang, Z. Ahmad, and J.S. Li, Magnetic-responsive microparticles with customized porosity for drug delivery, Rsc. Adv. 6 (2016) 88157-88167.

[59] S. Soltani, M. Ebrahimian-Hosseinabadi, and A.Z. Kharazi, Chitosan/graphene and $\operatorname{poly}(\mathrm{D}, \mathrm{L}-\mathrm{lactic}-\mathrm{co}$-glycolic acid)/graphene nano-composites for nerve tissue engineering, Tissue. Eng. Regen. Med. 13 (2016)684-690.

[60] K.J. Lynch, O. Skalli, and F. Sabri, Investigation of surface topography and stiffness on adhesion and neurites extension of PC12 cells on crosslinked silica aerogel substrates, Plos. One. 12 (2017)185978.

[61] J.E. Trachtenberg, J.K. Placone, B.T. Smith, C.M. Piard, M. Santoro, D.W. Scott, J.P. Fisher, and A.G. Mikos, Extrusion-Based 3D Printing of Poly(propylene fumarate) in a Full-Factorial Design, Acs. Biomater. Sci. Eng. 2 (2016)17711780 .

[62] J.C. Wang, H. Zheng, M.W. Chang, Z. Ahmad, and J.S. Li, Preparation of active $3 \mathrm{D}$ film patches via aligned fiber electrohydrodynamic (EHD) printing, Sci. Rep. 7 (2017)43924.

[63] J.C. Wang, M.W. Chang, Z. Ahmad, and J.S. Li, Fabrication of patterned polymer-antibiotic composite fibers via electrohydrodynamic (EHD) printing, J. Drug Deliv. Sci. 35 (2016)114-123. 
[64] Y. Gao, Y. Bai, D. Zhao, M.W. Chang, and J.S. Li, Tuning Microparticle Porosity during Single Needle Electrospraying Synthesis via a Non-SolventBased Physicochemical Approach, Polymers. 7 (2015)2701-2710.

[65] S. Ramesh, Y.T. Fung, and S. Chia Jun, Conductivity and FTIR studies on PEOLiX [X: CF3SO3(-), SO4(2-)] polymer electrolytes, Spectrochim. Acta. A. 69 (2008)670-675.

[66] X. Li, Y.M. Cao, G.D. Kang, H.J. Yu, and Z.N. Liu, Preparation of Antimicrobial Nanofiltration Membrane via Self-polymerization of Dopamine and Surface Grafting of PHGH, Chem. J. Chinese. U. 35 (2014)2026-2030.

[67] K. Ren, Y. Wang, T. Sun, W. Yue, and H. Zhang, Electrospun PCL/gelatin composite nanofiber structures for effective guided bone regeneration membranes, Mat. Sci. Eng. C-Mater. 78 (2017)324-332.

[68] T.I. Horuz and K.B. Belibagli, Nanoencapsulation by electrospinning to improve stability and water solubility of carotenoids extracted from tomato peels, Food. Chem. 268 (2018)86-93.

[69] V. Tucureanu, A. Matei, and A.M. Avram, FTIR Spectroscopy for Carbon Family Study, Crit. Rev. Anal. Chem. 46 (2016)502-520.

[70] M. Sattary, M.T. Khorasani, M. Rafienia, and H.S. Rozve, Incorporation of nanohydroxyapatite and vitamin D3 into electrospun PCL/Gelatin scaffolds: The influence on the physical and chemical properties and cell behavior for bone tissue engineering, Polym. Advan. Technol. 29 (2017)451-462.

[71] P. Mehdi, H. Marie-Claude, and A. Abdellah, Core-shell structured PEOchitosan nanofibers by coaxial electrospinning, Biomacromolecules. 13 (2012) 412-421.

[72] F. Abd Hamid, F.M. Salleh, N.S. Mohamed, and S. Adnan, The Effect of Graphene Content on the Structure and Conductivity of Cellulose/Graphene Composite, Sains. Malays. 46 (2017)1025-1031.

[73] C.Z. Liao, Y.C. Li, and S.C. Tjong, Graphene Nanomaterials: Synthesis, Biocompatibility, and Cytotoxicity, Int. J. Mol. Sci. 19 (2018)3564.

[74] D.M. Bigg, Mechanical-Properties of Particulate Filled Polymers, Polym. Composite. 8 (1987)115-122.

[75] B. Gangil, V. Kukshal, A. Sharma, A. Patnaik, and S. Kumar, Development of Hybrid Fiber Reinforced Functionally Graded Polymer Composites for Mechanical and Wear Analysis, Advances in Polymer Composites: Mechanics, Characterization and Applications 2057 (2019).

[76] H.X. Wang, M. Domingos, and F. Scenini, Advanced mechanical and thermal characterization of 3D bioextruded poly(e-caprolactone)-based composites, Rapid. Prototyping. J. 24 (2018)731-738.

[77] Z.C. Yao, S.C. Chen, Z. Ahmad, J. Huang, M.W. Chang, and J.S. Li, Essential Oil Bioactive Fibrous Membranes Prepared via Coaxial Electrospinning, J. Food. Sci. 82 (2017)1412-1422.

[78] Z.X. Meng, W. Zheng, L. Li, and Y.F. Zheng, Fabrication and characterization of three-dimensional nanofiber membrane of PCL-MWCNTs by electrospinning, Mat. Sci. Eng. C-Mater. 30 (2010)1014-1021. 
[79] H.M. Powell and S.T. Boyce, Engineered Human Skin Fabricated Using Electrospun Collagen-PCL Blends: Morphogenesis and Mechanical Properties, Tissue.Eng. Part. A. 15 (2009)2177-2187.

[80] S.J. Yan, X. Zhang, L.G. Zhang, H. Liu, X.F. Wang, and Q. Li, Polymer scaffolds for vascular tissue engineering fabricated by combined electrospinning and hot embossing, Biomed. Mater. 13 (2018)12.

[81] I. Lasocka, L. Szulc-Dabrowska, M. Skibniewski, E. Skibniewska, W. Strupinski, I. Pasternak, H. Kmiec, and P. Kowalczyk, Biocompatibility of pristine graphene monolayer: Scaffold for fibroblasts, Toxicol. In. Vitro. 48 (2018)276-285.

[82] V.M. Merkle, P.L. Tran, M. Hutchinson, K.R. Ammann, K. DeCook, X.Y. Wu, and M.J. Slepian, Core-shell PVA/gelatin electrospun nanofibers promote human umbilical vein endothelial cell and smooth muscle cell proliferation and migration, Acta. Biomater. 27 (2015)77-87.

[83] J.H. Lee, Y.J. Lee, H.J. Cho, and H. Shin, Guidance of In Vitro Migration of Human Mesenchymal Stem Cells and In Vivo Guided Bone Regeneration Using Aligned Electrospun Fibers, Tissue.Eng. Part. A. 20 (2014)2031-2042. 